\title{
Lasers as a Bridge between Atomic and Nuclear Physics
}

\author{
Sergei Matinyan \\ Duke University, Physics Department \\ and \\ Yerevan Physics Institute, Armenia
}

July 30, 2018

\begin{abstract}
This paper reviews the application of optical and UV laser radiation to several topics in low-energy nuclear physics. We consider the laser-induced nuclear anti-Stokes transitions, the laser-assisted and the laser-induced internal conversion, and the Electron Bridge and Inverse Electron Bridge mechanisms as tools for deexciting and exciting of low-lying nuclear isomeric states. A study of the anomalous, by low-lying, nuclear isomeric states (on an example of the ${ }^{229}$ Th nucleus) is presented in detail.
\end{abstract}

PACS: 21.10.-k, 23.20.Lv, 23.20.Nx, 32.80.-t, 32.80.Wr, 42.62.-b

Keywords: laser-assisted and laser-induced nuclear transitions; Electron Bridge and Inverse Electron Bridge mechanisms; excitation and deexcitation of nuclear levels; nuclear isomers; anomalously low-lying nuclear levels. 


\section{Contents}

$\begin{array}{lr}\text { Preface } & 4\end{array}$

\begin{tabular}{lll}
\hline & Introduction & 6
\end{tabular}

1.1 Simple estimate . . . . . . . . . . . . . . . . 6

1.2 Nuclear Compton Effect . . . . . . . . . . . . . . . . . . . . 7

2 Atomic Shells in the "Compton" Excitation of Nuclei 8

\begin{tabular}{|ll|}
\hline 3 & Laser induced nuclear anti-Stokes transitions \\
\hline
\end{tabular}

$3.1 \quad \beta$-transitions of nuclei . . . . . . . . . . . . . . . . . 12

3.2 Electromagnetic transitions of nuclei . . . . . . . . . . . . . . 13

\begin{tabular}{|lll}
\hline 4 & Laser-assisted internal conversion & 17
\end{tabular}

4.1 Theoretical background . . . . . . . . . . . . . . . . . . . . . . 17

4.2 Some examples: $N$ vs. 1 . . . . . . . . . . . . . . . . . . . 24

4.3 Experimental problems . . . . . . . . . . . . . . . . 25

5 Electron Bridge mechanism as a source of deexcitation of $\begin{array}{ll}\text { nuclei } & 27\end{array}$

$5.1 \quad$ Experimental observation . . . . . . . . . . . . . 27

5.2 The role of lasers in the EB mechanism of deexcitation of nuclei 29

5.3 Theoretical considerations . . . . . . . . . . . 30

$5.4 \quad$ Inverse Electron Bridge mechanism of nuclear excitation . . . 36

5.5 Some estimates . . . . . . . . . . . . . . . . 37

6 Lasers in the study of anomalously low-lying isomeric nuclear states: $\left.\left({ }^{229 m} T h\right)\right] 39$

6.1 Introductory remarks . . . . . . . . . . . . . . . . 39

6.2 Deexcitation of isomeric state by EB mechanism . . . . . . . . 41

6.3 Pumping isomers by laser resonance . . . . . . . . . . . . . . . . . . . . 43

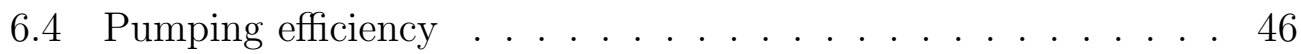

6.5 Modified IEB Mechanism in the study of ${ }^{229 m} \mathrm{Th}$. . . . . . . . 48

\begin{tabular}{llll}
\hline 7 & Summary and conclusions & 51
\end{tabular}

Appendix A: Parameters characterizing the interaction of the laser radiation with electron (dimensional arguments) 54 
Appendix B: Two representations of the interaction of the radiation with matter

Notation Index

60

References

62 


\section{Preface}

The present paper reviews the direct application of laser radiation to several topics in nuclear physics.

Here the term "direct" refers to the use of laser radiation in the optical and UV region (energy of the laser photons $\hbar \omega_{L} \sim 1-10 \mathrm{eV}\left(\lambda_{L}=10^{-4}-10^{-5}\right.$ $\mathrm{cm})$ ) for studying low-energy nuclear phenomena and related processes.

The "traditional" approach of using laser radiation in nuclear physics is well known. The Compton backscattering of laser photons off accelerated electrons produces intense, highly $(\sim 100 \%)$ polarized $\gamma$-ray beams with excellent energy resolution. This very effective method of employing conventional lasers in particle and nuclear physics was proposed long ago [1] and was implemented successfully in various accelerator facilities (SLAC, BNL, LURE (Orsay), ETL (Tsukuba)). The recent completion of the Free Electron Laser (FEL) Facility at Duke University gives possibility to yield $\gamma$-ray fluxes of $\approx 10^{7}-10^{9} \frac{\gamma}{s}$ (a factor of $10^{3}$ higher than is obtainable with conventional lasers in the energy range from 2-200 MeV [2] for studying phenomena of low and intermediate energy nuclear and particle physics.

In the present survey these kinds of topics in Nuclear Physics will be completely avoided. The aim here is to consider the wide range of very lowenergy nuclear physics topics where the "direct" (i.e., not transformed into $\gamma$-rays by Compton backscattering) laser beam! interaction with electronic atomic shells serves as a tool for studying the properties of low-lying nuclear levels and accompanying dynamical processes.

The theoretical study of these phenomena has a long and sometimes controversial history. It is interesting to point out that the history of these studies dates back to the 1920's when Einstein discussed the possibility that radioactivity could be induced by the action of optical quanta [3].

The literature on the subject of the present $\overline{\text { review }}$ is comprised of more than one hundred studies published mostly in American and former Soviet physical journals. To my knowledge there has not been an attempt to review this field exhaustively. Unfortunately, in the review literature, the main papers from the former USSR, which contribute significantly to the field, are not discussed appropriately. The short review paper [4] suffers from onesidedness.

\footnotetext{
${ }^{1}$ For the qualitative features of the interaction of the laser radiation with electron see Appendix A.
} 
Motivated by these reasons and stimulated by some of my colleagues from Triangle Universities Nuclear Laboratory (TUNL) and the Physics Department of Duke University, I present a rather complete review of the field covering the "direct" use of laser radiation in the energy range of the FEL Facility at Duke University $\left(E_{L} \lesssim 12 \mathrm{eV}\right)$ for studying low-energy nuclear physics phenomena.

Due to the diversity of the topics and methods, the present survey has to be rather schematic, but this will be compensated for by providing a detailed list of references.

The paper is organized as follows:

After introductory remarks concerning the ineffectiveness of laser radiation in the optical range for the unmediated study of properties of nuclei, the role of the atomic shell as a mediator is shown in the case of Compton excitation of nuclei. In Section 3, the laser-induced nuclear anti-Stokes transitions are investigated and electromagnetic transitions are compared with $\beta$-transitions. It is shown that the laser-induced electromagnetic anti-Stokes transitions are effective only for very strong laser fields $\left(I \gtrsim 10^{19} \frac{\mathrm{W}}{\mathrm{cm}^{2}}\right)$. Section 4 is devoted to the laser-assisted internal conversion process. The laser plays an important role in specific nuclei where the internal conversion in the laser-free case is forbidden by energy conservation. The technique for calculating the internal conversion coefficient for this process is presented. Experimental issues related to this process are discussed. In Section 5 we study the Electron Bridge mechanism as a means for deexciting nuclei and we discuss the role of laser radiation in enhancing the deexcitation. The Inverse Electron Bridge mechanism is presented as a tool for exciting nuclear states to their isomeric states. Section 6 is devoted to the study of the anomalously low-lying nuclear isomeric states in the ${ }^{229 m}$ Th nucleus. Methods introduced in Sections 4 and 5 are used for the detailed study of this phenomenon. Finally, Section 7 gives a summary.

In Appendix A dimensional arguments are used to give some useful information about the role that the high intensity plays in the interaction of radiation with electrons and atoms. Finally, Appendix B deals with the important issue of two alternative ways of describing the interaction of radiation with matter. 


\section{Introduction}

Q. Is it possible to obtain induced radioactivity by bombardment of matter with quanta of light?

A. First of all, I have to say that, probably, there exists radioactivity of matter induced by the action of the light quanta; the difficulty of the observation of such phenomenon, if it exists, is that the effect which has to be observed is very small. The confirmation of this effect is hard but possible ? $^{2}$

\subsection{Simple estimate}

Since the wavelength of the laser radiation $\lambda_{L}$ is much larger than the nuclear size $R_{A}\left[\sim(2-10) \times 10^{-13} \mathrm{~cm}\right]$, the direct coupling strength of laser photons to a nuclear system considered as a quantum state with level spacing $\Delta E_{A}$ of the order of one hundred $\mathrm{keV}$, is very small, even at extremely high radiation intensity. It is worthwhile, to appreciate this smallness, to make the following simple estimate. The interaction of electromagnetic radiation with a nucleus is more conveniently described by the Hamiltonian $]^{\text {in }}$ the so-called GöppertMayer's gauge 5

$$
H_{L A}=-e \sum_{p=1}^{Z} \overrightarrow{r_{p}} \cdot \overrightarrow{E_{L}}(t)
$$

where $\overrightarrow{r_{p}}$ is the radius vector of individual protons in the nucleus and $\overrightarrow{E_{L}}$ is

\footnotetext{
${ }^{2}$ On April 16, 1925, the National Academy of Science of Argentina held a special session to award A. Einstein the Academy's honorary member diploma.

At the reception, Einstein answered the questions of the Academy members. Four years later, details of the Session and the "interview" were published in the Proceedings of the Academy (Reception de la doctor Alberto Einstein en la session especial de la Akademia el dia 16 de april de 1925; Annales Sociedad Cientifica Argentina, 107, 337-347 (1929)) [3].

${ }^{3}$ The problem of relating the dipole from (1) to the commonly used form of the matterfield interaction Hamiltonian $\frac{1}{2 m}(\vec{p}-e \vec{A})^{2} \rightarrow-\frac{e}{m} \vec{p} \cdot \vec{A}+\frac{e^{2}}{2 m} \vec{A}^{2}$, has a long and controversial history which is resolved in the paper of Ref. [6]. See Appendix C for some details.
} 
the electric field of the laser radiation. $\left|\overrightarrow{E_{L}}\right|$ is proportional to $I^{\frac{1}{2}}$ where $\mathrm{I}$ is the laser beam intensity which will be measured hereafter in units of $\frac{W}{\mathrm{~cm}^{2}}$.

¿From (1) the following estimate for the magnitude of the matrix element can be derived:

$$
\mathbf{M} \approx\left(10^{-12}-10^{-13}\right) I^{\frac{1}{2}} \mathrm{eV} .
$$

Using for the nuclear level spacing $\Delta E_{A} \gtrsim 1 \mathrm{keV}$, we obtain for the coupling strength in the best case scenario (e.g., when the shielding of the atomic shells is ignored)

$$
\frac{\mathbf{M}}{\Delta E_{A}} \lesssim\left(10^{-15}-10^{-16}\right) I^{\frac{1}{2}}
$$

The situation for the direct use of laser radiation remains hopeless even in the case of very low lying levels (e.g., for ${ }^{229} T h$, where $\Delta E_{A}$ is on the order of a few eV. See Section 6).

\subsection{Nuclear Compton Effect}

Here we will show that the direct excitation of nuclear levels by X-rays or by hypothetical $\gamma$-ray lasers does not look encouraging either.

Consider, for example, the nuclear excitation by the Compton effect at resonance (See Fig. 1 for the corresponding Feynman diagrams).

For the cross section we have in the one resonance level approximation:

$$
\sigma_{\gamma \gamma}^{A}=\frac{C_{N}}{k_{L}^{2}} \frac{\Gamma_{I N} \Gamma_{N F}}{\left(E_{I N}-\hbar \omega_{L}\right)^{2}+\frac{\Gamma^{2}}{4}},
$$

where $\Gamma_{I N}$ and $\Gamma_{N F}$ are the widths of level $N$ associated with the transition to the initial state, $I$, and to the final state, $F$, respectively. Here, $k_{L}\left(\omega_{L}\right)$ is the wave number (frequency) of the incident laser photon, $C_{N}$ is the statistical weight of the intermediate state $N$, and $\Gamma$ is the total width of the level $N$. $E_{I N} \equiv E_{I}-E_{N}$, where $E_{I(N)}$ is the energy of the state $I(N)$.

Estimates [7] with $E_{F}-E_{I}-\hbar \omega_{L} \approx 10^{5} \mathrm{eV}$ give for the cross section

$$
\sigma_{\gamma \gamma}^{A} \approx 10^{-39}-10^{-40} \mathrm{~cm}^{2}
$$

which practically is unobservably small.

These introductory examples show the ineffectiveness of direct excitation of nuclear levels by radiation. 


\section{Atomic Shells in the "Compton" Excita- tion of Nuclei}

The example considered in the Introduction suggests the following modification to the Compton mechanism involving the excitation of low-lying nuclear levels: when the Compton effect occurs on the electron bound in the atomic shell, excitation of the nucleus takes place instead of scattering of the photon.

The mechanism is described by the diagrams in Figs. 2a and $2 \mathrm{~b}$. We note that if one exchanges the direction of the photon line (absorption of a photon is changed to emission of a photon in the final state) one arrives at the so-called internal Compton effect where the photon from the deexcitation of the nucleus is scattered on a bound electron.

This last mechanism is the basis of the Electron Bridge (EB) mechanism whereas the first one (described by the diagrams in Fig. 2) corresponds to the Inverse Electron Bridge (IEB) mechanism. The EB mechanism can be considered as alternative to the well-known Internal Conversion (IC) process where the deexcitation of the nucleus results in ejection of an electron out of the atomic shell without emission of a photon.

We will see below how laser radiation can assist in influencing the EB and IC mechanisms by resonating at the corresponding atomic level or by eliminating the mismatch between the nuclear $(\Delta E)$ and atomic $(\Delta \epsilon)$ level differences.

Here, two aspects of laser radiation are very important:

i) The dipole character of the interaction with the electronic shell which, of course, is common to all kinds of electromagnetic radiation. This property leads to an effective reduction of possible multipolarities inherent, in general, to nuclear radiation;

ii) unique, due to the lasers' very high intensity, is the possibility of multiphoton absorption (emission). This fact facilitates the resonance matching between $\Delta E$ and $\Delta \epsilon$.

These two essential aspects of laser radiation will be focused on below in the study of the role of laser radiation in low energy nuclear physics.

Turning now to the excitation of low-lying nuclear states (generally non-

resonant) by $\gamma$-rays (diagrams in Figs. $2 \mathrm{a}$ and $2 \mathrm{~b}$ ), we write the matrix elements $\mathbf{M}_{\mathbf{a}}$ and $\mathbf{M}_{\mathbf{b}}$ corresponding to Figs. $2 \mathrm{a}$ and $2 \mathrm{~b}$ : 


$$
\begin{aligned}
& \mathbf{M}_{a}=\sum_{n} \frac{\left\langle f F\left|H_{i n t}\right| I n\right\rangle\left\langle n\left|H_{\gamma}\right| i\right\rangle}{\epsilon_{n}-\epsilon_{i}-\hbar \omega_{L}} \\
& \mathbf{M}_{b}=\sum_{n} \frac{\left\langle f\left|H_{\gamma}\right| n\right\rangle\left\langle n F\left|H_{i n t}\right| i I\right\rangle}{\epsilon_{n}-\epsilon_{f}+\hbar \omega_{L}}
\end{aligned}
$$

We have used the following notations: $|i\rangle,|n\rangle,|f\rangle$ describe the initial, intermediate, and final states of an electron with energies $\epsilon_{i}, \epsilon_{n}, \epsilon_{f}$ respectively. $I$ and $F$ denote the initial and final states of the nucleus with energies $E_{I}$ and $E_{F} . H_{\text {int }}$ is the Hamiltonian describing the interaction of an electron with a nucleus and $H_{\gamma}$ is the Hamiltonian describing the interaction of a photon with an electron.

Conservation of energy gives: $\epsilon_{i}+\hbar \omega_{L}=\epsilon_{f}+E_{F I}\left(E_{F I} \equiv E_{F}-E_{I}\right)$.

We confine ourselves here to the non-covariant perturbation theory $(\operatorname{see}(5 \mathrm{a}, \mathrm{b}))$ since the exact calculation requires construction of the relativistic Green's function of an electron in the field of an atom. The physically simpler treatment above is transparent and effective.

We concentrate on the case where the laser photon energy is much smaller than the rest energy of the electron, $\hbar \omega_{L}<m c^{2}$ or, more precisely, smaller than the electron binding energy in the shell, $\hbar \omega_{L} \lesssim(\alpha Z)^{2} m c^{2}$, where $\alpha=\frac{e^{2}}{\hbar c}$ is the fine structure constant, $\mathrm{m}$ is the electron mass, and $\mathrm{Z}$ is the atomic number.

Inspection of the denominators in the matrix elements $(5 \mathrm{a}, \mathrm{b})$ shows that the main contribution to $\mathbf{M}_{\mathbf{a}}$ results from the summation over positive energy $\epsilon_{n}$ values of the states $|n\rangle$, whereas the main contribution to $\mathbf{M}_{\mathbf{b}}$ comes from negative values of $\epsilon_{n}$. That means that the excitation of the nucleus described by diagram 5a occurs mainly through inelastic scattering of a free electron $\left(\epsilon_{n}>0\right)$ (free-free transitions). Therefore, diagram 5 a gives the main contribution at sufficiently high incident photon energies $\left(\hbar \omega_{L} \gg(\alpha Z)^{2} m c^{2}\right)$. On the other hand, diagram $5 \mathrm{~b}$ is more important when the nucleus is excited in transitions of the electron between bound-bound and bound-free atomic states. This indicates that this diagram gives the main contribution at the energies we are interested in $\left(\hbar \omega_{L} \lesssim(\alpha Z)^{2} m c^{2}\right)$. Calculations [7] indeed confirm these physical reasonings. 
In the calculation of the cross section

$$
\sigma_{\gamma}=\frac{2 \pi}{\hbar} \frac{1}{2 J_{I}+1} \sum_{i} \frac{1}{2 J_{i}+1} \sum_{m_{i} m_{f}} \sum_{m_{I} m_{F}}\left|M_{b}\right|^{2} \rho\left(\epsilon_{f}\right)
$$

(here $\rho\left(\epsilon_{f}\right)$ is the density of the states of the final electron, $m_{i}, m_{f}$ and $m_{I}, m_{F}$ are magnetic quantum numbers of the initial and final states of the electron and the nucleus, respectively; the summation index $i$ denotes the occupied states in the (initial) electron shell) the approximation of one resonance level $n$ [7] is used which is more effective and reasonable in the case of bound-bound transitions. If the energies of the nuclear excitation and of the electronic transition are sufficiently close, a significant increase of the cross section is achievable.

The resulting cross section includes the probability of $\gamma$-radiation of multipolarity $L$ emitted by the nucleus, and the well-known matrix elements for the electronic electromagnetic transitions $\mathbf{M}(E L)$ and $\mathbf{M}(M L)$ ( $E$ and $M$ in $\mathbf{M}$ refer to electric and magnetic transitions, respectively). For higher multipolarities, the cross section drops sharply $\left(\sim\left(\frac{R_{A}}{a}\right)^{2 L}\right.$, where $R_{A}$ is the radius of the nucleus and $a$ is the radius of the electron shell). Thus, the maximum value of $\sigma_{\gamma}$ occurs for transitions with the lowest possible multipolarity $L$. Expressions for the matrix elements $\mathbf{M}(E(M), L)$ are given in the literature (see, e.g. 88]). The summation in (6) is carried out over the occupied states in the initial and final electron shells, and the choice of the characteristics of the intermediate state $|n\rangle$ (e.g., its angular momentum $j_{n}$, etc.) depends on the initial state $|i\rangle$. In the calculations the widths of the atomic and nuclear levels are usually neglected in comparison with the parameter $\Delta \equiv E_{I}-E_{F}+\epsilon_{n}-\epsilon_{i}$. This parameter enters in the cross section in the form $\left[\frac{\left(E_{I}-E_{F}\right)}{\Delta}\right]^{2}$. ¿From this, it is evident that for the bound-free transition case, $\epsilon_{i}<\epsilon_{n}, \Delta \approx E_{I}-E_{F}$, i.e., the resonance condition is not fulfilled.

The largest cross section occurs for the bound-bound atomic transitions. This case is most effective if $E_{I F}$ is sufficiently close to the energy of the electronic transition ( $\Delta$ is small). $M 1$ transitions are very important. For example, the cross section of the Compton excitation of the isotype ${ }_{50}^{110} \mathrm{Sn}$ with the $M 1$ transition $\frac{1}{2}^{+} \rightarrow \frac{3}{2}^{+}, E_{F I}=24 \mathrm{keV}$, the atomic transition $2 s_{\frac{1}{2}} \rightarrow 1 s_{\frac{1}{2}}$ and $\Delta=0.74 \mathrm{keV}$, one obtains $\sigma_{\gamma}=4 \mu b$. In Table I, we show the parameters for nuclear and atomic transitions and the resulting cross sections $\sigma_{\gamma}$ for several long-lived isotopes. The energies of the nuclear transitions have been taken from [9] and those of the electronic transitions from [10]. We see that the magnitudes of the cross section are considerable $\left(\sim\left(10-10^{2}\right) \mu b\right)$. 
The case of the bound-free transitions is less effective. Here, $\sigma_{\gamma}$ is of the order of $\left(10^{-2}-1\right) \mu b$. For example [7]:

${ }_{26}^{57} \mathrm{Fe}, \sigma_{\gamma}=5 \cdot 10^{-2} \mu b ; \quad{ }_{36}^{83} \mathrm{Kr}, \sigma_{\gamma}=10^{-1} \mu b ; \quad{ }_{54}^{129} \mathrm{Xe}, \sigma_{\gamma}=1 \mu b$.

The conclusion from the above considerations is that the relatively low lying nuclear vibrational, collective states can be excited at a rate of $\left(10^{-7}-10^{-9}\right)$ per photon.

\begin{tabular}{|c|c|c|c|c|c|c|}
\hline Isotope & $E_{F I}, \mathrm{keV}$ & $J_{I} \rightarrow J_{F}$ & $j_{i} \rightarrow j_{n}$ & $\epsilon_{j_{n} j_{i}}, \mathrm{keV}$ & $\Delta, \mathrm{keV}$ & $\sigma_{\gamma}, \mu b$ \\
\hline${ }_{50}^{119} \mathrm{Sn}$ & 24 & $\frac{1}{2}^{+} \rightarrow \frac{3}{2}^{+}$ & $2 s_{\frac{1}{2}} \rightarrow 1 s_{\frac{1}{2}}$ & 24.74 & 0.74 & 4 \\
\hline${ }_{53}^{129} \mathrm{~J}$ & 27 & $\frac{7}{2}^{+} \rightarrow \frac{5}{2}^{+}$ & $2 s_{\frac{1}{2}} \rightarrow 1 s_{\frac{1}{2}}$ & 27.88 & 0.88 & 5 \\
\hline${ }_{52}^{125} \mathrm{Te}$ & 36.5 & $\frac{1}{2}^{+} \rightarrow \frac{3}{2}^{+}$ & $3 s_{\frac{1}{2}} \rightarrow 1 s_{\frac{1}{2}}$ & 30.81 & -4.7 & 0.14 \\
\hline${ }_{66}^{161} \mathrm{Dy}$ & 43.8 & $\frac{1}{2}^{+} \rightarrow \frac{3}{2}^{+}$ & $2 s_{\frac{1}{2}} \rightarrow 1 s_{\frac{1}{2}}$ & 44.74 & 0.44 & 110 \\
\hline${ }_{76}^{185} \mathrm{Tm}$ & 8.42 & $\frac{1}{2}^{+} \rightarrow \frac{3}{2}^{+}$ & $3 s_{\frac{1}{2}} \rightarrow 2 s_{\frac{1}{2}}$ & 7.81 & -0.61 & 196 \\
\hline${ }_{76}^{189} \mathrm{Os}$ & 69.60 & $\frac{3}{2}^{-} \rightarrow \frac{5}{2}^{-}$ & $3 s_{\frac{1}{2}} \rightarrow 1 s_{\frac{1}{2}}$ & 70.82 & 1.22 & 26 \\
\hline${ }_{77}^{193} \mathrm{Ir}$ & 73.1 & $\frac{3}{2}^{+} \rightarrow \frac{1}{2}^{+}$ & $3 s_{\frac{1}{2}} \rightarrow 1 s_{\frac{1}{2}}$ & 72.85 & -0.25 & 9 \\
\hline${ }_{79}^{197} \mathrm{Au}$ & 77 & $\frac{3}{2}^{+} \rightarrow \frac{1}{2}^{+}$ & $3 s_{\frac{1}{2}} \rightarrow 1 s_{\frac{1}{2}}$ & 77.4 & 0.4 & 10 \\
\hline${ }_{80}^{201} \mathrm{Mg}$ & 1.57 & $\frac{3}{2}^{-} \rightarrow \frac{5}{2}^{-}$ & $2 p_{\frac{3}{2}} \rightarrow 2 p_{\frac{1}{2}}$ & 1.93 & 0.36 & 17 \\
\hline${ }_{78}^{193} \mathrm{Pt}$ & 12.7 & $\frac{1}{2}^{-} \rightarrow \frac{3}{2}^{-}$ & $4 s_{\frac{1}{2}} \rightarrow 2 s_{\frac{1}{2}}$ & 13.04 & 0.34 & 62 \\
\hline
\end{tabular}

Table I. Parameters of nuclear $\left(E_{F I}(\mathrm{keV}), J_{I} \rightarrow J_{F}\right)$ and atomic $\left(\epsilon_{j_{n}, j_{i}}\right.$, $\left.j_{i} \rightarrow j_{n}\right)$ bound-bound transitions, $\Delta=E_{I}-E_{F}+\epsilon_{j}-\epsilon_{j_{i}}=-E_{F I}+\epsilon_{j_{n} j_{i}}$, and calculated Compton excitation cross sections for some long-lived isotopes [7].

Of course, the experimental situation is very difficult, due to the high background associated with the intense primary and secondary fluxes of Xrays. The fact that the energies of the $\mathrm{X}$-rays and the nuclear radiations do not coincide (the difference reaches several keV) can perhaps be used to detect and to study low-lying states in nuclei. Concluding, we mention for the sake of completeness, that in the case of higher energy $\gamma$-rays $\left(\hbar \omega_{L} \gg(\alpha Z)^{2} m c^{2}\right)$, where diagram 5a gives the main contribution, the cross sections are much lower, typically of the order of $10^{-34} \mathrm{~cm}^{2}$.

The above examples which do not always have a direct connection with the nuclear physics conducted by UV and optical lasers (the main topic of interest in the present work), teaches us that atomic physics is merged with 
nuclear physics due to the coupling of electromagnetic radiation with the electronic shells. Lasers with their high intensity have the potential to enhance and sometimes induce nuclear (weak and electromagnetic) transitions.

Below we will illustrate these issues in several examples (Sections 3-6).

\section{Laser induced nuclear anti-Stokes transi- tions}

\section{$3.1 \beta$-transitions of nuclei}

Lasers, like other sources of electromagnetic radiation, provide the possibility of enhancing transitions from excited nuclear states and of obtaining information on specific nuclear transitions unaccessible otherwise.

Consider, as an example, a three-level nuclear system as illustrated in Fig. 3. First, we concentrate on the $\beta$-transition: The nucleus initially is in the isomeric state $a$ from which it $\beta$-decays (very slowly) to its ground state $b$ with the rate $\gamma_{a b}^{w}$. Under the influence of an external electromagnetic field with frequency $\omega_{L}$ one can induce a two-step $\beta$-decay of $a$ to $b$ : a virtual excitation of the nucleus to the level $c$ by absorption of a single (or, sometimes multiple) photon with energy $\hbar \omega_{L}$ and rate $\gamma_{c a}^{e l}$ of the laser field and subsequent $\beta$-decay from $c \rightarrow b$ with rate $\gamma_{c b}^{w}$.

This scheme gives not only the possibility of increasing the rate of the $\beta$-decay to the state $b$ (if $\gamma_{c b}^{w}>\gamma_{a b}^{w}$; of course, $\gamma_{c b}^{w} \ll \gamma_{c a}^{e l}$ ) but provides the information on the $\beta$-transition $c \rightarrow b$ which is sometimes not accessible [11].

Before turning to the more interesting cases where all the transitions discussed above are of electromagnetic nature, thus eliminating the condition $\gamma_{c b}^{w} \ll \gamma_{c a}^{e l}$ (unavoidable for the $\beta$-transitions) it is worth mentioning that claims (1977-1983) about strong modifications of forbidden and even allowed $\beta$-decay total rates [12 by very intense, long-wavelength, coherent electromagnetic radiation were not confirmed, although their discussion has attracted the interest of many authors. The latest investigations (1987) have demonstrated that there is no laser field-dependent enhancement of the total $\beta$-decay rates in the long-wavelength limit, although the spectra are greatly modified (for a more complete review and the resolution of the controversies in some previous studies see [13, 14]). However, we note that, in principle, the rate of another nuclear $\beta$-process, the orbital electron capture by a nucleus (so called K-capture) is naturally influenced by the laser radiation, because 
the probability of this process depends on the electron density in the vicinity of the nucleus. Therefore, the K-capture process can be sensitive to the influence of a strong electromangetic field. Particularly, in contrast to the case of the $\beta$-decay where the total rate does not depend on the nuclear spin orientation f for the orbital electron capture, due to the hyperfine interaction between the electron and the nucleus, the total rate of K-capture depends critically on the orientation of the electron and nuclear spins, and therefore, on temperature [15].

The rate of the orbital electron capture from an unfilled $s$ orbital of a free atom or ion at low temperature $(k T \ll \delta$, where $\delta$ is the hyperfine splitting) depends on the sign of the hyperfine constant $h$ [15]. The application of a laser field with the frequency $\omega_{L}=\frac{\delta}{\hbar}$ can influence the rate. In the particular case of negative $h$, when the orbital electron capture practically does not take place, it is possible to induce this process by applying the resonant radiation with $\omega_{L}=\frac{\delta}{\hbar}$. Large effects are expected especially for hydrogen-like ions since the competing decays from other bound electrons are absent. In principle, properly chosen radiation could cause a modulation of the electron capture rate and the subsequent characteristic X-ray emission could serve as a monitor for observing this process.

As a justification of the old wisdom that "the novel is well forgotten olden" and for the sake of completeness, we note that a very similar idea was proposed almost two decades ago [16] for modifiying the population of hyperfine structure sub-levels of $\mu$-mesic atoms through resonant laser radiation, and the subsequent change in the nuclear $\mu$-capture probability.

\subsection{Electromagnetic transitions of nuclei}

Turning now to the case of electromagnetic nuclear transitions, we again consider the three-level nuclear system with electromagnetic transition rates $\gamma_{a b}, \gamma_{c a}$, and $\gamma_{c b}$ and an applied laser field of frequency $\omega_{L}$ to promote the $a \rightarrow c$ transition (Fig. 4) from the isomeric state ${ }^{m} a$ to the level $c$.

It is easy to recognize that this scheme resembles the scheme of the fa-

\footnotetext{
${ }^{4}$ Of course, due to the parity non-conservation, the probability of the particle $\left(e^{-}\right.$or $\nu_{e}$ ) emission parallel to the nuclear spin in $\beta$ decay differs from the probability for the emission in the opposite direction. However, the total rate does not depend on nuclear spin orientation simply because of the isotropy of space (rotational invariance).

${ }^{5}$ The orbital electron capture probability rapidly decreases with an increase in the number of the competing atomic shells from which an electron can be captured.
} 
mous Lamb-Retherford experiment on the Lamb shift measurement of the hydrogen atom where the level a corresponds to the $2 s_{\frac{1}{2}}$ atomic level, the level $b$ corresponds to the level of $1 s_{\frac{1}{2}}$ ground state, and level $c$ corresponds to the $2 p_{\frac{1}{2}}$ state which is excited by electron beam bombardment.

The fact that, in contrast to the $\beta$-decay, $\gamma_{c a} \approx \gamma_{c b}$, opens new possibilities. The state $c$ can decay to states $a$ and $b$ by the emission of radiation and also by internal conversion (IC) to other, not specified states with total decay rate $\gamma_{c}=\gamma_{c a}+\gamma_{c b}+\gamma_{I C}+\ldots$.

We take the interaction between the external radiation field and the system from (1) with $\vec{E}_{L}=\vec{E}_{0} \cos \omega_{L} t$. The laser field can be considered homogeneous (spatially uniform) as its wavelength is much larger than atomic (and, of course, nuclear) dimensions.

The equations of motion for the probability amplitudes of states $a$ and $c$ in the interaction representation (so called, Bethe-Lamb equations) 117, 18, 19, 6] are :

$$
\begin{gathered}
\dot{a}=-\frac{1}{2} \gamma_{a b} a-\frac{i}{2 \hbar} V_{c a} e^{-i \Delta t} \\
\dot{c}=-\frac{1}{2} \gamma_{c} c-\frac{i}{2 \hbar} V_{a c} e^{i \Delta t}
\end{gathered}
$$

where $V_{a c}$ is the transition dipole matrix element between the states $a$ and $c$, and $\Delta=\frac{E_{c}-E_{a}}{\hbar}-\omega_{L}$ is a detuning frequency. In the following, we concentrate on the most interesting case where the spontaneous decay of state $c$ to the isomeric state $a$ is negligible $\left(\gamma_{c a} \approx 0\right.$, no backcoupling $\left.c \rightarrow a\right)$.

Solving then Eqs. (7) with the initial conditions $a(0)=1, c(0)=0$, we have:

$$
\begin{gathered}
|a(t)|^{2}=e^{-\gamma_{a b} t} \\
|c(t)|^{2}=\left(\frac{\left|V_{a c}\right|}{2 \hbar}\right)^{2} \frac{e^{-\gamma_{a b} t}+e^{-\gamma_{c} t}-2 e^{-\gamma t} \cos \Delta t}{\Delta^{2}+\delta^{2}}
\end{gathered}
$$

with

$$
\left(\begin{array}{l}
\gamma \\
\delta
\end{array}\right)=\frac{\left(\gamma_{a b} \pm \gamma_{c}\right)}{2}
$$


Since we are interested in the case where the state $c$ decays much faster than the isomeric state $a\left(\gamma_{c} \gg \gamma_{a b}\right), \gamma=-\delta=\frac{\gamma_{c}}{2}$. We see that the population of the level $c$ decays, to the first order of the dipole interaction, on the same time scale $\gamma_{a b}^{-1}$ as $|a(t)|^{2}$.

This is an anti-Stokes process since the frequency $\omega_{c b}$ of the transition to state $b$ is increased. The rate of this anti-Stokes process is defined as the product of $|c(t)|^{2}$ and the rate $\gamma_{c b}$ for the transition $c \rightarrow b$.

Calculating the total probability $N_{\gamma}^{a S}(T)$ of this process for the time interval $T$ (duration of the laser pulse) smaller than the lifetime of the isomer $a\left(T \ll \gamma_{a b}^{-1}\right)$ and larger than the lifetime of the intermediate state $c$ $\left(\frac{1}{\gamma_{c}} \ll T \ll \frac{1}{\gamma_{a b}}\right)$, and dividing it by the probability $N_{\gamma}^{s p}(T)$ of the spontaneous decay $a \rightarrow b$ during the same time interval, one obtains for the ratio

$$
\eta=\frac{N_{\gamma}^{a S}(T)}{N_{\gamma}^{s p}(T)}
$$

the expression [19]

$$
\eta=\left(\frac{\left|V_{a c}\right|^{2}}{2 \hbar}\right)^{2} \frac{\gamma_{c b}}{\gamma_{a b}} \frac{1}{\Delta^{2}+\frac{\gamma_{c}^{2}}{4}} .
$$

For the matrix element $V_{a c}$ in (10), assuming that the nucleus is spinless, one finds

$$
\left|V_{a c}\right|^{2}=\frac{1}{3}\left(e E_{0}\right)^{2}|\langle c|\vec{r}| a\rangle|^{2}
$$

with $E_{0}^{2}=\frac{4 \pi \hbar}{\lambda_{L}} \phi$, where $\phi$ is the photon flux and $\lambda_{L}$ is the wavelength of the radiation.

The nuclear dipole matrix element $\langle c|\vec{r}| a\rangle$ can be expressed by the lifetime $\gamma_{c a}^{-1}$ using a semiclassical calculation of Einstein's A-coefficient in the condition of the thermal equilibrium [18]. The resulting relation coincides with the result of the quantum mechanical Weisskopf-Wigner theory for the spontaneous decay [20]:

$$
\left|V_{a c}\right|^{2}=\frac{\hbar}{2 \pi} \frac{\lambda_{c a}^{3}}{\lambda_{L}} \Gamma_{c a} \phi
$$


with $\lambda_{c a}=\frac{2 \pi \hbar c}{E_{c}-E_{a}}$ and the width $\Gamma_{c a}=\hbar \gamma_{c a}$ for the transition $c \rightarrow a$. Finally, one obtains for $\eta$ :

$$
\eta=\frac{\hbar \phi}{\Gamma_{a b}}\left(\frac{\lambda_{c a}}{\lambda_{c}}\right)^{3} \frac{\lambda_{L}^{2}}{8 \pi} \frac{\Gamma_{c b} \Gamma_{c a}}{(\hbar \Delta)^{2}+\frac{\Gamma_{c}^{2}}{4}}
$$

where we introduced, instead of the rates $\gamma$, the corresponding widths, $\Gamma=$ $\hbar \gamma$.

The factor $\left(\frac{\lambda_{c a}}{\lambda_{c}}\right)^{3}$ in (13), which has been left out of the first theoretical study of this process, makes the experimental situation very difficult. For instance, at laser photon energies of $\hbar \omega_{L}=10 \mathrm{eV}$ and a nuclear transition level difference $E_{c}-E_{a}=100 \mathrm{keV}$ this factor is $10^{-12}$, which leads to very small $\eta$ values for moderate laser intensities. The effect is noticeable only for small nuclear level spacings $\left(\lesssim 10-10^{-2} \mathrm{eV}\right)$. The situation can be improved using resonant laser radiation with $\hbar \Delta \ll \frac{\Gamma_{c}}{2}$. As can be seen from (10), in this case and under the conditions of moderate laser intensities $\left(\left|V_{a c}\right|<\Gamma_{c}\right.$, but $\left|V_{a c}\right| \gtrsim\left(\Gamma_{c} \Gamma_{a b}\right)^{\frac{1}{2}}$, i.e., $\left.I \gtrsim 10^{14} \frac{W}{c m^{2}}\right) \eta$ can be of the order of unity. This means that the rates of two-step and one-step processes are equal. For higher intensities $\left(\left|V_{a c}\right|>\Gamma_{c}\right)$ the anti-Stokes process dominates. This condition corresponds to $I \gtrsim 10^{19} \frac{\mathrm{W}}{\mathrm{cm}^{2}}$ (see [19] for details). Under these conditions, Eqs. (7) must be solved precisely. The resulting picture looks as follows (again $\Delta \ll \frac{\gamma_{c}}{2}, \gamma_{a b} \ll \gamma_{c}$ ). The system oscillates between the levels $a$ and $c$. In contrast to the weak field case $\left(\left|V_{a c}\right| \ll \frac{\Gamma_{c}}{2}\right)$, where level $c$, as well as $a$, decays with the slower rate $\gamma_{a b}$, now this laser-excited state decays with the faster rate $\gamma \approx \frac{\gamma_{c}}{2}$. The long lived isomer $a$ is depleted via $c$ to $b$ on a very short time scale. For the case of the isomeric state ${ }^{111 m} \mathrm{Pd}$ one obtains $\gamma_{c}^{-1} \approx 1.5 \cdot 10^{-11} \mathrm{~s}\left(\Gamma_{c a}=2.3 \cdot 10^{-6} \mathrm{eV}(\mathrm{E} 1\right.$ transition $), \Gamma_{c b}=4.3 \cdot 10^{-5} \mathrm{eV}(\mathrm{E} 2$ transition), $\left.\Gamma_{c} \approx 4.5 \cdot 10^{-5} \mathrm{eV}\right)$. The condition $\left|V_{a c}\right| \gtrsim \Gamma_{c}$ corresponds to the intensity $I \gtrsim 1.3 \cdot 10^{19} \frac{\mathrm{W}}{\mathrm{cm}^{2}}$. The total probability for the anti-Stokes process in a very strong field approaches its maximal value

$$
N_{j}^{a S}(T)=\frac{\Gamma_{c b}}{2 \Gamma_{c}} \frac{\left|V_{a c}\right|^{2}}{\Gamma_{a b} \Gamma_{c}+\left|V_{a c}\right|^{2}} \approx \frac{\Gamma_{c b}}{2 \Gamma_{c}}, \quad T \gg \frac{1}{\gamma_{c}}
$$

Hence, a pulse of $\approx 0.1 \mathrm{~ns}$ with the above intensity ensures that all irradiated isomeric nucleus decay. The main experimental problem with the resonant excitation is the severe condition $\hbar|\Delta| \ll \frac{\Gamma_{c}}{2}$ which imposes very 
strong constraints on the bandwidth $\frac{\Delta \omega}{\omega}$ of the radiation necessary for $\eta$ to exhibit a resonant peak. The associated requirements on the beam monochromaticity can be somewhat compensated for at the expense of high intensity. It is important to note that the theoretical treatment with only a single intermediate atomic state is reliable only very close to the resonance.

Concluding this section, we notice that similar results to those obtained here in the case of a strong radiation field $\left(\left|V_{a c}\right| \gtrsim \Gamma_{c}\right)$ apply to the nuclear $\beta$-decay |11] where, as mentioned above, $\gamma_{c a}$ is always much larger than $\gamma_{c b}$. For the $\gamma$-transitions of nuclei one can find nuclei with $\gamma_{c a} \ll \gamma_{c b}$. In this specific case the spontaneous decay of the intermediate state $c$ tends not to go back to the isomeric state $a$ but leads into the final ground state $b$.

\section{Laser-assisted internal conversion}

\subsection{Theoretical background}

Internal conversion (IC) is a clear example where nuclear physics (deexcitation of a nucleus) is merged with an atomic process (ejection of the electron from an atomic shell). Thus, in the spirit of the present review, it is quite appropriate to consider IC in detail, especially because the theoretical approaches developed for studying laser assisted IC are common to some other nuclear-atomic processes where lasers play an important role.

In this process, as already pointed out in the Introduction, the effective reduction of the multipolarity of nuclear gamma-ray transitions plays a crucial role. However, there exists another issue where lasers have a substantial influence: changing the atomic surroundings of a nucleus which affects IC (we already encountered this kind of laser influence in Section 3 when we considered the role of lasers in the orbital electron capture). The influence of a laser field on IC has been investigated firstly just from this point of view 21.

For weakly bound electrons participating in the IC, the laser-assisted removal of one of the electrons that significantly contribute to IC leads to a significant decrease of the coefficient of IC. We consider here the more

\footnotetext{
${ }^{6}$ The coefficient of IC $\alpha_{I C}$ is defined as the ratio of the probability $W_{I C}$ for IC and the radiative transition probability $W_{\gamma}$ for a given nuclear transition $E(M) L$ with electric (magnetic) radiation multipolarity $L$. IC coefficients can be calculated by well developed methods [8].
} 
direct and efficient role of the intense laser radiation field on the IC process. In general, if the IC process is non-zero in the laser free case $\left(\alpha_{I C}^{\text {free }} \neq 0\right)$ the change of $\alpha_{I C}$ due to the laser influence is small $\left(\alpha_{I C}^{f r e e} \gg \alpha_{I C}^{L A}\right)$. This is obvious, in principle, because the energy of the laser photons under study $(\lesssim 10 \mathrm{eV})$ is generally small in comparison with the energy $E_{\gamma}=E_{I F}=$ $E_{I}-E_{F}$ of the nuclear transition. (Below, we will consider the exception from this general rule in the case of low-lying nuclear levels.) However, in nuclei where the energy difference $E_{I F}=E_{\gamma}$ is less than the magnitude of the electron binding energy $E_{B}\left(\Delta=E_{\gamma}-\left|E_{B}\right|<0\right)$, IC does not take place $\left(\alpha_{I C}^{\text {free }}=0\right)$. If the photon energy of the laser beam $\hbar \omega_{L}$ is of the order of $|\Delta|$ the originally forbidden IC process may occur via absorption of the necessary numbers of laser quanta. Thus, the electromagnetic radiation (laser) essentially accelerates the nuclear $\gamma$-decay rate and, more importantly, induces IC which is absent otherwise.

We first consider some theoretical issues connected with the treatment of the laser-assisted IC. The theoretical description of the electronic states under the joint action of the Coulomb potential of the nucleus and the intense radiation field is only approximate; no precise analytical solution is known. We confine ourselves here to a simplified non-relativistic model [23, 24, 25, 26, 27 for the system "nucleus + electron + intense radiation field" with Hamiltonian] $H=H_{o}+H_{I}$, where $H_{o}=H_{o N}+H_{o e}$ is the sum of the Hamiltonians for the unperturbed nucleus $H_{o N}$ (the explicit form of $H_{o N}$ does not enter in the following treatment) and the single electron $H_{o e}$ participating in $\mathrm{IC}$,

$$
H_{o e}=-\frac{Z e^{2}}{R}+\frac{1}{2 m}\left(\vec{p}-\frac{e}{c} \vec{A}\right)^{2}
$$

Here, $\vec{A}$ is the vector potential of the external laser field which will be specified later; $\vec{R}, \vec{p}$, m denote electron coordinates, momentum and mass, respectively. The interaction between the nucleus and electron is of the Coulomb type

$$
H_{I}=-\sum_{p=1}^{Z} \frac{e^{2}}{\left|\vec{R}-\overrightarrow{x_{p}}\right|}+\frac{Z e^{2}}{R}
$$

\footnotetext{
${ }^{7}$ There are several other approximate models in the literature, e.g., the model where the Coulomb potential of the nucleus is replaced by a spherical harmonic oscillator 22.
} 
where $\overrightarrow{x_{p}}$ denotes the nuclear p-th proton radius vector. Relations (15) and (16) reflect a strong simplification, first of all, because the interaction between the electron and the intense radiation field is comparable to the binding energy of the electrons of the atomic shell. However, for the inner electrons (K, L-shells), the Coulomb potential of the nucleus still dominates and the modification of the initial electron states by the laser field can be treated perturbatively. For the final (free) electrons one can use the well-known Volkov solution for the charged particle in the electromagnetic field [28]. Furthermore, since in this model one only uses (16) for the electron-nucleus interaction [29], the photon exchange between the nucleus and the electron shell does not appear explicitly. Such a simplified treatment emphasizes the corrections which at least are necessary to improve the theory of this process:

i) use of the relativistic Dirac Hamiltonian for the bound electronic state "dressed" by the intense radiation field. This improvement requires mainly numerical calculations.

ii) taking into account the effect of the nuclear size, and

iii) the screening of the Coulomb potential of the nucleus by inner-shell electron clouds.

Item i) is most important and has no exact analytic solution (and the same holds for $(15),(16))$.

Thus, approximations are unavoidable, and we will be confined here to the simplifications described above. Since the final free electron is influenced by the intense laser field, the corresponding wave function is based on Volkov's solution of the Dirac [28 and Klein-Gordon 30 equations for an electromagnetic plane wave with specially chosen initial conditions.

Volkov's paper has not received much attention for a long time, presumably due to the absence of appropriate radiation sources at that time. However, with the invention of the laser, the theoretical interest has been renewed, and different exact and approximate solutions have been given with various choices of the initial conditions and different methods for the theoretical description (classical relativistic and non-relativistic, quantum mechanical and quantum electrodynamical [31, 32, 33, 34, 35]). We give here the original Volkov solution for the Klein-Gordon (KG) and Dirac(D) equations. With the gauge for the radiation field vector potential $A_{0}(x)=0$, we have: 


$$
\begin{aligned}
\psi_{K G}(x) & =e^{-i p \cdot x} \exp \left[-\frac{i e}{2 p \cdot q} \int_{-\infty}^{\beta} d \beta^{\prime}\left[2 \vec{p} \vec{A}\left(\beta^{\prime}\right)-e \vec{A}^{2}\left(\beta^{\prime}\right)\right]\right] \\
\psi_{D}(x) & =\psi_{K G}(x)\left[1+e \frac{\vec{q} \cdot \vec{A}}{2 p \cdot q}\right] u(p),
\end{aligned}
$$

where $p(\epsilon, \vec{p})$ is the four-momentum of the charged particle, $q(\omega, \vec{q})$ is one of the laser photons, $u(p)$ is a Dirac spinor, and $\beta \equiv q \cdot x=\omega t-\vec{q} \cdot \vec{x}$. The scalar product $p \cdot q=\epsilon \omega\left(1-\frac{\vec{v} \cdot \vec{q}}{\mid \vec{q})}\right)$ is in the nonrelativistic case $(v \ll 1)$ simply given by $m \omega$, and $\psi_{D}(x) \approx \psi_{K G}(x) u(p)$, and finally $p \gg e A=\frac{e E_{0}}{\omega}$, where $E_{0}$ is the amplitude of the laser electric field.

Under these conditions, the initial and final electronic states appear as two different approximations of the solution of the Hamiltonian (16):

$$
\begin{gathered}
\psi_{i}(\vec{R}, t)=\phi_{n j \lambda}(\vec{R}) e^{\frac{-i E_{B} t}{\hbar}} \exp \left(\frac{i e}{\hbar c} \vec{A} \cdot \vec{R}\right) \\
\psi_{f}=\exp \left[-\frac{i}{2 m \hbar} \int^{t}\left[\vec{p}-\frac{e}{c} \vec{A}\left(t^{\prime}\right)\right]^{2} d t^{\prime}\right] u_{c}^{(-)}(\vec{R})
\end{gathered}
$$

Here $\phi_{n j \lambda}(\vec{R})$ is a hydrogen-type solution with quantum numbers $n, j, \lambda$ and energy eigenvalue $E_{B}$ and $u_{C}^{(-)}(\vec{R})$ is an electron Coulomb wave function. In relations (18) and (19) we restored, temporarily, $\hbar$ and $c$.

For the vector potential of the radiation field we write for the circular (c) and linear $(\ell)$ polarization:

$$
A_{\ell}=\left(\begin{array}{c}
a\left[\hat{e}_{1} \cos \beta+\hat{e}_{2} \sin \beta\right] \\
a \hat{e}_{3} \cos \beta
\end{array}\right)
$$

The unit vectors $\hat{e}_{1}, \hat{e}_{2}, \hat{e}_{3}$, perpendicular to each other, define the frame of reference, and $a=\frac{c E_{0}}{\omega}$.

Inspection of (18) and (19) shows that if we add to the condition $p \gg$ $\frac{e E_{0}}{\omega}=\frac{e E_{0} \lambda_{L}}{2 \pi c}$ (where the momentum $p=\hbar k(k$ - wave number)) the condition $\frac{e}{\hbar c} \vec{A} \cdot \vec{R} \ll 1$, which is well satisfied for the inner shells, we achieve a great 
simplification: i) the space-dependent and time-dependent parts of the Smatrix element of the process can be separated, ii) the space-dependent part is the same as in the laser-free case.

Notice that the first condition $\left(p \gg \frac{e E_{0} \lambda_{L}}{2 \pi c}\right)$ can be rewritten as the condition that the kinetic energy of the final electron is larger than $\frac{r_{0} I \lambda^{2}}{2 \pi^{2}} \approx$ $10^{-14} I \lambda_{L}^{2} \approx 10^{-26} I \lambda_{L}^{2} \mathrm{eV}$ where $r_{0}$ is the classical radius of the electron, and the laser intensity $I$ is measured in $\frac{W}{\mathrm{~cm}^{2}}$ and $\lambda_{L}$ in $\mathrm{cm}$. Thus, this number is extremely small $\left(\lambda_{L} \sim 10^{-5} \mathrm{~cm}\right)$ for any reasonable intensity. (See Appendix $\mathrm{B}$ for the dimensional analysis of scales characterizing the laser interaction with the electron.) Using the above approximation, one is able to calculate the laser induced IC coefficient $\alpha_{n j \lambda}^{L, l a s}$ for a transition of multipolarity L and for an electronic state of quantum numbers $n, j, \lambda$. The calculations include the point nucleus approximation $\left(\left|\vec{x}_{p}\right|<|\vec{R}|\right)$, the expansion $\frac{1}{\left|\vec{R}-\vec{x}_{p}\right|}$ of (16) in terms of spherical harmonics, and the Wigner-Eckart theorem which permits to express the matrix element of the electric multipole moment of order $\ell, m$ through its reduced matrix element between the nuclear states I and $\mathrm{F}$ [36]. Because this reduced matrix element decreases rapidly with increasing $\ell$, we are confined to the lowest $\ell$ determined by the multipolarity $L$ of the nuclear transition, as usual. The further evaluation involves the use of the addition theorem for spherical harmonics and the orthogonality of the $3 \mathrm{j}$ symbols that enter here, and the averaging (and summing) over the magnetic quantum numbers of the initial (final) nuclear and electronic states, etc.

The approximation of neglecting the screening of the nuclear Coulomb potential is improved somewhat by using instead of the charge $e Z$ the effective charge $e Z_{\text {eff }}(n)=e n\left(\frac{\left|E_{B}\right|}{R_{y}}\right)^{\frac{1}{2}}$, where $R_{y}=\frac{e^{2}}{2 a_{B}}, \mathrm{n}$ is the principal quantum number of the electronic state with binding energy $E_{B}$, and Bohr radius $a_{B}$. The effective charge $e Z_{\text {eff }}$ corresponds to the charge of the hydrogen-like nucleus where the electron has the same binding energy as in the real atom with the same quantum numbers.

As we emphasized above, we are interested in the situation where $\alpha_{I C}^{\text {free }}=$ 0 , i.e., $\Delta<0$. For our purposes, of course, the most important case is the case near threshold: $\hbar \omega_{I F} \lesssim\left|E_{B}\right|$. The laser field must be so intense that the interaction energy of the electrons with the laser field becomes comparable to the binding energy of the electrons in the shell. This gives a condition for the laser field intensity 


$$
I \geq\left(\frac{Z_{e f f}}{n}\right)^{4}\left[\hbar \omega_{L}(e V)\right]^{2} 6.31 \cdot 10^{8} \quad \text { in } \frac{W}{\mathrm{~cm}^{2}} .
$$

If one uses the small electron momentum assumption $\frac{p n a_{B}}{Z_{\text {eff }}} \ll 1$, then $|\Delta| \ll$ $\left|E_{B}\right|$.

The final result for $\alpha_{n j \lambda}^{L, l a s}$ depends on the following parameters: Nuclear (and atomic) parameters: $E_{\gamma}=E_{I}-E_{F}, L$ - multipolarity of the nuclear $\gamma$-rays, $Z_{\text {eff }}$, and $\Delta=E_{\gamma}-\left|E_{B}\right|$. The laser parameters enter in the following combinations: $r_{0}=\frac{\Delta}{\hbar \omega_{L}}, I$, and $r=r_{0}-\frac{2 e^{2} I}{m \hbar \omega_{L}^{3}}$. The second term in the parameter $r$ multiplied by $\hbar \omega_{L}$ gives the laser pondermotive potential which in some cases reduces the effectivity of the very high intensity $I$.

For the case $\Delta<0$, the $\alpha_{n j \lambda}^{L, l a s}$ is expressed in terms of the threshold IC coefficient $\alpha_{n j \lambda}^{L, T h}$ which is theoretically calculable. Thus, if $\hbar \omega_{L}$ is comparable to $|\Delta|$, then, for the laser-free forbidden case, IC can start after absorption of the corresponding number of laser photons demanded by energy conservation. As a result, we have 24,

$$
\alpha_{n j \lambda}^{L, l a s}=\alpha_{n j \lambda}^{L, T h} T\left(\begin{array}{l}
c \\
l
\end{array}\right)
$$

where

$$
T_{\left(\begin{array}{l}
c \\
l
\end{array}\right)}=\sum_{N>-r} T_{\left(\begin{array}{l}
c \\
l
\end{array}\right)}^{\left(b_{N}\right)}
$$

with index $c(l)$ corresponding to circular (linear) polarization. $N$ in $(26)$ is a positive integer. Here $b_{N}=b_{0}(N+r)^{\frac{1}{2}}$ with

$$
b_{0}=\frac{e E_{0}}{m \omega_{L}^{2}} \sqrt{\frac{\hbar \omega_{L}}{R_{y}}} \frac{1}{a_{B}}=1.07 \cdot 10^{-6}\left(\frac{I}{\left(\hbar \omega_{L}\right)^{3}}\right)^{\frac{1}{2}} .
$$

As before we measure $I$ in $\frac{W}{\mathrm{~cm}^{2}}$ and $\hbar \omega_{L}$ in $\mathrm{eV}$. The summation in (23) goes over the number of laser photons absorbed by the electronic shell. In (23), 


$$
T_{c}\left(b_{N}\right)=\frac{1}{2 b_{N}} \int_{0}^{b_{N}} J_{2 N}(x) d x,
$$

where $J_{2 N}(x)$ is the Bessel function. The corresponding formula for $T_{\ell}\left(b_{N}\right)$ includes the generalized Bessel functions $J_{N}(b, d)$ :

$$
T_{\ell}\left(b_{N}\right)=\int_{0}^{1} J_{N}^{2}\left(b_{N} x,-\frac{d}{4}\right) d x
$$

For weak laser fields $\left(b_{0}<1\right.$, or $\left.I<10^{12}(\hbar \omega)^{3}\right)$, applying the small argument expression for the Bessel and generalized Bessel functions, we obtain

$$
\begin{aligned}
& T_{c}=\left(\frac{b_{N_{\min }}}{2}\right)^{2 N_{\min }} \frac{\left(2 N_{\min }\right) ! !}{\left(N_{\min } !\right)^{2}\left(2 N_{\min }+1\right) ! !} \\
& T_{\ell}=\left(\frac{b_{N_{\min }}}{2}\right)^{2 N_{\min }} \frac{1}{\left(N_{\min } !\right)^{2}\left(2 N_{\min }+1\right)},
\end{aligned}
$$

where $N_{\min }$ is the minimum number of laser photons necessary for the onset of the originally forbidden IC process. As $b_{N}^{2} \sim I, T_{\left(\begin{array}{l}l \\ c\end{array}\right)} \sim I^{N_{\min }}$.

Curves exist in the literature [24] where the dependence of $T\left(b_{0}, r_{0}\right)((23)$, (25)) on the laser intensity is given for different materials and lasers with $I<10^{14} \frac{\mathrm{W}}{\mathrm{cm}^{2}}$. Unfortunately, for higher $I\left(\lesssim 10^{20} \frac{\mathrm{W}}{\mathrm{cm}^{2}}\right)$ such curves do not exist. It is important to note that for larger values of $I\left(>10^{14-16} \frac{\mathrm{W}}{\mathrm{cm}^{2}}\right)$ the hindering effect of the laser pondermotive potential [37] must be taken into account. This potential $\frac{2 e^{2} I}{m \hbar \omega_{L}^{3}} \cdot \hbar \omega_{L}\left(\approx 2 \mathrm{eV}\right.$ at $\left.I=10^{13} \frac{\mathrm{W}}{\mathrm{cm}^{2}}\right)$ that enters into the expression for $r=r_{0}-\frac{2 e^{2} I}{m \hbar \omega_{L}^{3}}$ leads to the necessity to increase $N_{\min }$ in the summation of (23) and, thus, decreases the probability of the process. Fig 5 shows the above mentioned curves for $T\left(b_{0}, r_{0}\right)$.

The "(anti) efficiency" of the pondermotive forces depends on the precise knowledge of the ionization thresholds of the electron states. The values for the ionization thresholds calculated in the nonrelativistic approximation can be changed appreciably in more precise calculations. The actual ionization threshold may be larger than the value suggested by the simplified model. 
Particularly, it was demonstrated 38] in a nonrelativistic calculation for the hydrogen atom that a laser field can keep the electron in the atom even at very high intensity. This phenomenon certainly needs more work to be understood. Recently, more refined calculations reduced the magnitude in the laser intensity [39]. Thus, a more detailed study of the ionization process and its effects on the electronic states and their widths is required.

\subsection{Some examples: $N$ vs. $I$}

Consider as an example for the above considerations 25 an isomeric E3 transition, ${ }^{105 m} \mathrm{Ag}$, with $E_{I F}=25.470 \mathrm{keV}$. The binding energy $\left|E_{B}\right|$ for the $\mathrm{K}$ shell is $25.524 \mathrm{keV}$ 40. Thus, $\Delta=-44 \mathrm{eV}$, and IC is forbidden for the laser-free case. In the model considered above it is possible to calculate the laser threshold IC coefficient $\alpha_{1 \frac{1}{2} 0}^{3 T h}=152$ [25].

The comparison of this calculation with the general, relativistic IC theory 41 leads to an agreement within $20 \%$.

For a laser with $\hbar \omega_{L}=6.42 \mathrm{eV}$, i.e., for the number of laser photons $N=7$ necessary to start IC, and $I=2.3 \cdot 10^{14} \frac{\mathrm{W}}{\mathrm{cm}^{2}}\left(b_{0}=1\right)$ one obtains $\alpha_{1 \frac{1}{2} 0}^{3 \text { Las }} \approx 10^{-13}$, an unobservably small value. For the hypothetical value of $\hbar \omega_{L}=30 \mathrm{eV}$ which would lead to $\mathrm{N}$ between 1 and 2 and with $I=2.4 \cdot 10^{16} \frac{\mathrm{W}}{\mathrm{cm}^{2}}$ we would have $\alpha_{1 \frac{1}{2} 0}^{3 I C}=0.16(N=2)$.

Consider now the two characteristic cases with $\Delta<0$ : the isomeric nuclei ${ }^{235 m} U$ and ${ }^{183 m} W$, for which atomic and nuclear data exist 40]. In Table II, the necessary data [40] and the calculated results for $\alpha_{n j \lambda}^{L}$ [24] are given. For ${ }^{235 m} U, E_{I F}=73.5 \mathrm{eV}$; for ${ }^{183 m} W, E_{I F}=544 \mathrm{eV}$. $\hbar \omega_{L}=5 \mathrm{eV}$ was taken for the laser photons. 


\begin{tabular}{|c|c|c|c|}
\hline Atom & \multicolumn{2}{|c|}{${ }^{235 m} U, E_{I F}=73.5 \mathrm{eV}$} & ${ }^{183 m} W, E_{I F}=544 \mathrm{eV}$ \\
\hline Electron Shell & $5 d_{\frac{3}{2}}\left(O_{4}\right)$ & $5 d_{\frac{5}{2}}\left(O_{5}\right)$ & $4 s_{\frac{1}{2}}\left(N_{1}\right)$ \\
\hline Binding Energy $\left|E_{B}\right|, \mathrm{eV}$ & 105 & 96 & 592 \\
\hline$\Delta=E_{I F}-\left|E_{B}\right|, \mathrm{eV}$ & -31.5 & -22.5 & -48 \\
\hline$\left.b=1.07 \cdot 10^{-6} \cdot \frac{n}{Z_{e f f}} \frac{I^{\frac{1}{2}}}{\left(\hbar \omega_{L}\right.}\right)^{\frac{3}{2}}$ & 0.55 & 0.58 & 0.44 \\
\hline$\beta_{0}$ & 5 & 5 & 9.5 \\
\hline$I, \frac{W}{c m^{2}}$ & $2.7 \cdot 10^{15}$ & $2.7 \cdot 10^{15}$ & $10^{16}$ \\
\hline $\mathrm{T}$ & 0.27 & 1.2 & 3.6 \\
\hline$\alpha_{n j \lambda}^{L, \text { las }}$ & $2.1 \cdot 10^{15}$ & $2.7 \cdot 10^{15}$ & $3.3 \cdot 10^{2}$ \\
\hline
\end{tabular}

Table II. Atomic and nuclear data and results of the calculations of $\alpha_{n j \lambda}^{L, l a s}$ 24] for $\hbar \omega_{L}=5 \mathrm{eV}, I=\beta_{0}^{2}\left(\hbar \omega_{L}\right)^{3} 8.73 \cdot 10^{11} \frac{\mathrm{W}}{\mathrm{cm} m^{2}}$, and $\beta_{0}=\frac{e E_{0}}{\omega_{L}} \sqrt{\frac{2}{\hbar \omega_{L} \cdot m c^{2}}}$.

The increase of the laser intensity (e.g., to $I=10^{20-21} \frac{\mathrm{W}}{\mathrm{cm} \mathrm{m}^{2}}$ ) will lead, for the near threshold cases, $\Delta<0$, to a drastic increase of the laser assisted IC coefficients $\alpha_{n j \lambda}^{L, l a s}$. The estimates lead to enormous values for $\alpha_{n j \lambda}^{L a s}$. Unfortunately, these estimates did not properly take into account the role of laser pondermotive forces with their hindering role at very intense fields. The detailed and accurate study at very high laser intensity will unavoidably demand the aid of modern, powerful computers.

Before turning to the experimental issues related to laser assisted (and induced) IC we would like to mention that via the electron bridge mechanism it is also possible to accelerate the IC process and to obtain higher values for the IC coefficients. This subject will be considered in Section 5 which is devoted to the study of this mechanism.

\subsection{Experimental problems}

The main problems faced in experimental studies of laser assisted and induced IC are the production and collection of a sufficient amount of isomeric nuclei and their irradiation by intense $\left(I>10^{14} \frac{\mathrm{W}}{\mathrm{cm}^{2}}\right)$ and relatively long laser pulses. Typically, one experiences a high background of ions and especially, electrons. Due to the high electron background, it may be more useful to detect the soft $\mathrm{X}$-rays which are emitted in the recombination processes when the vacancies 
left by the conversion electrons are occupied by electrons from higher shells, rather than to detect the slow IC electrons.

The number of emitted soft X-ray photons $N_{\gamma}$ can be estimated as

$$
N_{\gamma}=\xi A t \frac{\alpha_{I C}^{l a s}}{\alpha_{I C}^{T}}
$$

where $\alpha_{I C}^{T}$ is the total laser free IC coefficient, $\alpha_{I C}^{\text {las }}$ is the laser induced IC coefficient for the shell under study (see Table II), $A$ is the activity of the sample, $t$ is the total irradiation time (duration of the laser pulse in an ordinary case), and $\xi$ is the efficiency of soft X-ray detection.

Taking, e.g., $\frac{\alpha^{l a s}}{\alpha^{T}} \approx 0.2$ (for ${ }^{183 m} W, \alpha^{\text {las }}=3.3 \cdot 10^{2}$, see Table II), $\xi=10^{-2}$, $t \approx 10^{-9} \mathrm{~s}$, we obtain the necessary activity to obtain $N_{\gamma} \approx 1$ in one laser pulse $A \approx 6 \cdot 10^{11} \mathrm{~Bq}(15 \mathrm{Ci}) .\left(1 \mathrm{C} i=3.7 \cdot 10^{10} \mathrm{~Bq}=3.7 \cdot 10^{10}\right.$ disintegrations per second.) The problem of producing a sample of low density but high activity can be solved using a method similar to the one proposed in 42 to sort out the isomeric nuclei embedded in molecules, e.g., in our case, $\mathrm{WO}_{3}$ [24]. This method uses the fact that some isomeric nuclei have angular momenta higher than the ground state which leads to different molecular spectra due to the difference in fine interactions.

The ${ }^{183 m} W$ isomer can be produced by thermal neutron capture: ${ }^{182} W(n, \gamma)$ ${ }^{183 m} W(\sigma \sim 2 m b)$ (the abundance of ${ }^{182} W$ is $\left.26.3 \%\right)$. Besides the fast isomer separation one needs a soft X-ray detection method of high enough resolution in order to be able to select soft X-rays from the recombination of the atomic shells $\left(N_{1}, N_{2}, N_{3}\right.$ in the case of $\left.{ }^{183 m} \mathrm{~W}\right)$.

Finally, the irradiation time $t$ may be increased to force the laser light to run back and forth through the sample multiple times, while a laser active material compensates for the losses, thus keeping the intensity approximately constant.

Concluding this section, we notice that the specific IC process, where the shell electron remains in the discrete state, can be considered also from the point of view of the Electron Bridge mechanism which we will study in the next section. Thus, the laser's role in the elimination of the mismatch between atomic and nuclear level differences (resonant IC) will be clearly seen. 


\section{Electron Bridge mechanism as a source of deexcitation of nuclei}

\subsection{Experimental observation}

A systematic way to use the electron shell as a mediator between the laser beam and the nucleus for studying nuclear low-energy properties is via the so called Electron Bridge (EB) mechanism.

The idea was born long ago, in 1958, in the former USSR 443 and has been developed more recently into a useful tool 44, 45]. The EB mechanism effectively transfers the energy of a nuclear transition to the atomic shell which, passing through excited intermediate state(s), emits monoenergetic $\gamma$-rays, thus providing the nuclear deexcitation. It is a 3rd order process with respect to the electromagnetic interaction (see Fig. 6 for the corresponding Feynman diagram). The energy of the emitted $\gamma$-rays $E_{\gamma}$ is $E_{I F}-\left(\epsilon_{f}-\epsilon_{i}\right)$ with $\epsilon_{i(f)}=-B_{i(f)}$, where $B_{i(f)}$ is the binding energy of the electron in the atomic shell $|i\rangle(|f\rangle)$. When $B_{i}>B_{f}\left(\epsilon_{f}>\epsilon_{i}\right), E_{\gamma}<E_{I F} \equiv E_{I}-E_{F}$, one obtains the so called "Stokes line" in the deexcitation spectrum.

The inverse EB (denoted as IEB below) mechanism corresponds to the analogous diagrams shown in Fig. 7 with the external radiation absorbed by the electron of the initially unperturbed shell, thus, providing a way to excite nuclei.

The first experimental observation of the nuclear deexcitation via an EB mechanism was done in 1985 by the Zagreb-Ottawa group [46]. In [46 the deexcitation of the $30.7 \mathrm{keV}$ (lifetime $T_{\frac{1}{2}} \sim 13.6 \mathrm{yr}$ ) isomeric level of ${ }^{93} \mathrm{Nb}$ was studied and $28.2 \mathrm{keV}$ energy photons were observed as a result of the $\mathrm{EB}$ mechanism involving the initial L-electron states (with binding energies $\left.B_{L_{1}}=2.675 \mathrm{keV}, B_{L_{2}}=2.426 \mathrm{keV}, B_{L_{3}}=2.368 \mathrm{keV}\right)$ and the final state $N_{5}\left(B_{N_{5}}<30 \mathrm{eV}\right)$ or higher electron states. The higher $\mathrm{L}$ states were used instead of the $\mathrm{K}$ state, since excitation of the $\mathrm{K}$ shell gives photons with energies below the niobium K X-ray energy, and the background is too high.

It is necessary to point out that the EB processes are usually accompanied by processes connected with IC channels which, as a second order process,

\footnotetext{
${ }^{8}$ As it was already stressed in the pioneering papers [43] on the EB mechanism, such third order effects in $\alpha$ should be substantial if the IC coefficients are very large. That reflects the close connection between the IC process (with an electron in the continuum, or in the discrete state) and the EB mechanism where an additional photon is emitted.
} 
is more probable, in general, than the 3rd order process under study (e.g., the external bremsstrahlung associated with IC of the $30.7 \mathrm{keV}$ level).

There are also other contributions from the internal Compton scattering produced in the deexcitation of the initial nuclear level, etc. However, in [46, these sources of background were found to be negligible.

Fig. 7 shows a spectrum of $\gamma$-rays after background subtraction. The first theoretical estimate given by the authors of 446] involves the numerical solution of the inhomogeneous Dirac equation for an electron in the nuclear Coulomb field and hydrogen-like atomic wave functions. The ratio $\eta$ calculated for the probability $W_{\gamma}^{(3)}$ of the 3rd order transition based on the diagrams Fig. 6 and the probability $W_{\gamma}^{(1)}$ of the direct nuclear deexcitation was found to be $\eta_{t h}=\frac{W_{\gamma}^{(3)}}{W_{\gamma}^{(1)}}=0.19$, whereas the experimental result was $\eta=0.070 \pm 0.018$. Later, more detailed theoretical calculations gave $\eta_{t h}=$ 0.069. 47

There exists a second experimental result [48] based on the study of the decay of isomeric state ${ }^{193 m} \operatorname{Ir}$ with energy $80.27 \mathrm{keV}\left(T_{\frac{1}{2}}=10 \mathrm{~d}\right)$. This isomeric state was obtained in the irradiation of a $99 \%$ enriched sample of ${ }^{192} \mathrm{O} s$ by thermal neutrons via the chain ${ }^{192} \mathrm{Os} \stackrel{(n, \gamma)}{\rightarrow} 193 \mathrm{Os} \underset{T_{\frac{1}{2}}=30 \mathrm{~h}}{\stackrel{\beta(0.3 \%)}{\rightarrow}} 193 \mathrm{~m}$ Ir.

Experiments conducted under the conditions of high background associated with the IC channel $\left(\approx 10^{5} W_{\gamma}^{(3)}\right)$ gave for $\eta$ the value $\approx 0.21$.

Theoretical estimates for $\mathrm{L}$ electrons yielded $\eta=0.18$ which should be taken only as the lower bound due to the neglect of the additional contributions from higher shells $(\mathrm{M}, \mathrm{N}, \ldots)$. Estimates in [48] give for $\eta(\mathrm{M}, \mathrm{N}, \ldots) \sim$ 0.1-0.2. Again, more precise calculations are in agreement with the experimental value 0.21 [47].

Before turning to the role of lasers in the nuclear deexcitation process provided by the EB mechanism, it is worthwhile to note the following. We have already mentioned that the probability for IC increases with the effectiveness of the EB mechanism. In turn, the existence of the EB channel leads to a significant decrease in the IC coefficient with respect to its so-called "tabulated" value where the EB mechanism is not taken into account. For instance, for the isomeric state ${ }^{235 m} U$, the "tabulated" IC coefficient $\left(\alpha_{I C}(E 3) \approx 10^{19}\right)$ is reduced by a factor of $\approx 10^{5}$ times [47]. 


\subsection{The role of lasers in the EB mechanism of deexci- tation of nuclei}

Now we will discuss the laser-assisted EB process where an electron of an atom containing an isomeric nucleus absorbs the nuclear transition photon of energy $E_{\gamma}=E_{I F}$ and emits a number $\mathrm{N}$ of atomic transition photons, thus eliminating the mismatch between $E_{I F}$ and $\epsilon_{n i}$ (the energy difference between the intermediate and initial atomic states). The resonantly excited electronic states finally decay by X-ray emission.

The schematic diagram of Fig. 8 explains the process for the case of the emission of two photons. In this scheme, an electron from the initial shell $|i\rangle$ "dressed" by the intense laser field absorbs a nuclear radiation quantum with energy $E_{\gamma}$, emits two laser photons with energy $2 \hbar \omega_{L}$, and then occupies resonantly the intermediate state $|n\rangle$ with energy $\epsilon_{n}\left(\epsilon_{n}=-B_{n}\right.$, where $B_{n}$ is the binding energy of the electron in shell $|n\rangle)$, and finally decays to the final state $|f\rangle(=|i\rangle$ in this particular case $)$ with emission of an X-ray photon with energy $\hbar \omega_{x}$. Due to the dipole character of emission (absorption) of the laser quanta $(\ell=1)$, the laser also "transfers" angular momentum to effectively reduce the multipolarity $\mathrm{L}$ of the nuclear transition radiation to $\mathrm{L}=1$ ( $\mathrm{L}=$ 3 for the case of ${ }^{235 m} U$ considered below), thus enhancing the probability of the process.

Fig. 9 presents the Feynman diagrams describing this process. At resonance, the first diagram is dominating. We have:

$$
E_{I}-E_{F}=\epsilon_{n}-\epsilon_{i}+N \hbar \omega_{L}
$$

or, for the detuning $\Delta$,

$$
\Delta=E_{I}-E_{F}+\epsilon_{i}-\epsilon_{n}
$$

The well-known and theoretically studied case is the isomeric nucleus ${ }^{235 m} U$ with energy $E_{I}=73.5 \mathrm{eV}$ (angular momentum $J_{I}=\frac{1}{2}^{+}, T_{\frac{1}{2}}=26$ min) which has an E3 transition to the ground state $\mathrm{F}$ with $J_{F}=\frac{7}{2}^{-}$.

Among the numerous electronic orbits of the uranium atom we choose the orbit $6 s_{\frac{1}{2}}$ with binding energy $71 \mathrm{eV}\left(\epsilon_{i}=-71 \mathrm{eV}\right)$, which is closest to $E_{\gamma}$, as the initial electronic shell $|i\rangle$. We describe the complex electronic structure of the uranium atom in the one-electron approximation, i.e., we neglect the 
splitting of shells with definite principal quantum number. At resonance this approximation is appropriate.

As an intermediate atomic shell, we select the $8 s_{\frac{1}{2}}$ shell with binding energy $2.14 \mathrm{eV}\left(\epsilon_{n}=-2.14 \mathrm{eV}\right)$. Thus, the detuning $\Delta$ is $4.64 \mathrm{eV}$. The resonance will be achieved by two (four) laser photon emission with energy 2.32 (1.16) $\mathrm{eV}$, respectively. The final atomic one-electron state $|f\rangle$ is supposed to be the same as $|i\rangle$ [49]. All electronic states $(|i\rangle,|n\rangle,|f\rangle)$ are "dressed" states.

Due to the laser, not only the resonance condition is fulfilled, but the originally E3 $\gamma$ transition is converted into the emission of an E1 X-ray photon, thus increasing the rate of the X-ray emission significantly. The laser beam makes it possible to avoid the selection rules of X-ray emissions which are acting in the laser free case.

The reader may notice the arbitrariness in the selection of the intermediate state $|n\rangle$ and its energy $E_{n}$. However, the (assumed) value of $E_{n}$ is not so important for the phenomenon under study from the point of view that it is a small change, whereas the change of the order of the resonance (i.e., the number $\mathrm{N}$ of laser photons needed to fulfill the resonance conditions, $\left.\Delta=N \hbar \omega_{L}\right)$ is crucial.

\subsection{Theoretical considerations}

The theoretical study of EB processes is analogous to the calculations of the laser assisted IC which were presented in Section 4. The theoretical framework was developed in the papers [50, 49, 51, 26, 27, 52].

The study 49, 51 is based on the Hamiltonians $H_{0}$ and $H_{I}$ (15), (16), and we take as substitute for (15) the dipole form (1): The interaction of the linearly polarized laser field with the electron is described classically by a Hamiltonian of type (1) $H_{L e}=-e \vec{r} \cdot \vec{E}_{L}(t)$ and the interaction of the emitted (quantized) X-ray field is described by the same kind of Hamiltonian $H_{e x}=-e \vec{r} \cdot \vec{E}_{x}(t)$, where

$$
\begin{gathered}
\vec{E}_{L}(t)=\vec{E}_{0} \hat{\epsilon}_{3} \cos \omega_{L} t \\
\vec{E}_{x}(t)=i \sum_{\omega_{x}, \epsilon}\left(\frac{2 \pi \omega_{x}}{V}\right)^{\frac{1}{2}} \vec{\epsilon}\left(a e^{-i \omega_{x} t}-a^{+} e^{i \omega_{x} t}\right)
\end{gathered}
$$


Here $\omega_{x}$ and $\vec{\epsilon}$ are the frequency and unit polarization vector of the emitted radiation, $a$ and $a^{+}$are the annihilation and creation operators, and $V$ is the normalization volume, respectively.

Again, as before, the influence of the other electrons is taken into account by the use of the effective nuclear charge $Z_{\text {eff }}(n)$ in the one-electron wave functions. As before, due to the expression (16) that describes the electronnucleus interaction, photon exchange between the nucleus and the electron shell does not appear [29].

In the treatment of the joint influence of the nuclear Coulomb and laser fields on the dressed electron in the subshell $|n\rangle$, it is convenient to use parabolic coordinates [49] for the shell of principle quantum number $n, n=$ $n_{1}+n_{2}+|m|+1$, here $n_{1}, n_{2}$ are the parabolic quantum numbers [53], and $m$ is the magnetic quantum number.

The wave function describing the "dressed" electron state can approximately be written as 54

$$
\psi\left(n, n_{1}, n_{2}, m\right)=\phi_{n_{1} n_{2} m} \sum_{N=-\infty}^{+\infty} J_{N}\left(\lambda_{n_{1} n_{2}}\right) e^{-i\left(E_{n}+N \hbar \omega_{L}\right) \frac{t}{\hbar}}
$$

where $\phi_{n_{1} n_{2} m}$ is a hydrogen-type solution in parabolic coordinates, $J_{N}$ is a Bessel function of the first kind, $N$ is the number of absorbed or emitted laser photons, $E_{n}=\epsilon_{n}-\frac{i \Gamma_{n}}{2}$ is the complex energy of the intermediate electronic state $|n\rangle$ with its energy $\epsilon_{n}$ and width $\Gamma_{n}$, and finally the argument of the Bessel function is

$$
\lambda_{n_{1} n_{2}}=\frac{3 n\left(n_{1}-n_{2}\right) E_{0} e a_{B}}{Z_{e f f} \hbar \omega_{L}} .
$$

This solution is used as a basis for the construction of the electron Greens function and S-matrix for the process.

The further procedure is analogous to the one we described for IC (section 4). The initial and final state is taken as $i s$ - and $f s$-type, respectively, where $i(f)$ is the principal quantum number for the $|i\rangle(|f\rangle)$ state $R_{i(f) 0}(r) Y_{00}$. Here, $R_{i(f) 0}$ is the nonrelativistic radial part of the hydrogen-type solution of principal quantum number $i(f)$ and orbital angular momentum $\ell=0$, and $Y_{00}$ is the corresponding spherical harmonics. $Z_{\text {eff }}$ for the $|i\rangle$ state $(\mathrm{n}=6)$ is equal to $Z_{\text {eff }}(6)=13.71$, and for the $|n\rangle$ state $(\mathrm{n}=8), Z_{\text {eff }}(8)=3.17$. 
As a final result of this procedure, we obtain for the ratio $\eta=\frac{W_{f i}^{l a s}}{W_{f i}^{s p}}$ of the probability $W_{f i}^{\text {las }}$ of the laser assisted resonant EB mechanism and the probability $W_{f i}^{s p}$ of the spontaneous $\gamma$ decay [29] the following expression:

$$
\eta=\frac{2 e^{2}}{9} \frac{[(2 L-1) ! !]^{2}}{(L+1)(2 L+1)} \frac{\left|J_{n i}\right|^{2}\left|I_{L, n i}\right|^{2}}{\left(\Delta-N \hbar \omega_{L}\right)^{2}+\frac{\Gamma_{n}^{2}}{4}}\left[\frac{c}{\omega_{I F}}\right]^{2 L-2} F,
$$

where $\Gamma_{n}=\Gamma_{n 0}+\Gamma_{n f}$, and $\Gamma_{n 0}$ is the natural linewidth of the state $|n\rangle$ for the laser-free case and $\Gamma_{n f}$ is the laser field contribution to the power broadened linewidth,

$$
\begin{array}{r}
J_{n i}=\int d r r^{3} R_{i 0}\left(Z_{e f f}(i), r\right) R_{n 1}\left(Z_{e f f}(n), r\right) \\
I_{L, n i}=\int d r r^{1-L} R_{i 0}\left(Z_{e f f}(i), r\right) R_{n L}\left(Z_{e f f}(n), r\right) .
\end{array}
$$

For given values of $i=6, n=8, L=3, Z_{\text {eff }}(i), Z_{\text {eff }}(n)$ we have for $J_{n i}$ and $I_{L, n i}$

$$
J_{86}=-0.51 a_{B}, I_{3,86}=4.4 \cdot 10^{-5} \frac{1}{a_{B}^{4}}
$$

The quantity $\mathrm{F}$ in (36) is

$$
F=\sum_{K}\left(\frac{\omega_{x k}}{\omega_{I F}}\right)^{3} F_{K}=\sum_{K} f_{K}
$$

where $\omega_{x K}=\omega_{I F}-N \omega_{L}+K \omega_{L}$, and $K$ is an integer. The quantities $f_{K}$ are quadratic combinations of products of the Bessel function $J_{K}\left(\lambda_{n_{1} n_{2}}\right)$. $J_{K}\left(\lambda_{n_{1} n_{2}}\right)$ with the proper Clebsh-Gordon coefficients. $f_{K}$ are maximal at $K=0, \pm 1$.

Figs, 10,11 show the intensity $\mathrm{I}$, and the $\mathrm{K}$ dependence of $F_{K}\left(f_{K}\right)$ [51.

At the resonance $\left(\Delta=N \hbar \omega, N=2\right.$ or $4, \hbar \omega_{L}=2.32 \mathrm{eV}$ or $1.16 \mathrm{eV}$, respectively) and a intensity $I \approx 10^{12} \frac{\mathrm{W}}{\mathrm{cm}^{2}}$, with an estimated value of $\Gamma_{n} \approx$ $10^{-5} \mathrm{eV}$ (from a $2 p \rightarrow 1 s$ transition of the same energy) we obtain for the ${ }^{235} \mathrm{U}$ isomeric state 49 


$$
\eta \approx 6.7 \cdot 10^{9}
$$

If one takes into account the fact that the intense laser broadens the linewidth $\Gamma_{n f}$ [51], the magnitude of $\eta$ decreases but still remains of the order of $10^{9}$, leading to expectations that the affect is accessible to experimental study, though the IC coefficient is much, much higher. We note that above we concentrated on the special case of the bound-bound atomic transitions where the final electron shell is the same as the initial one $(|i\rangle=|f\rangle)$. In this case the IC strongly dominates over the EB process.

Concluding this part of our consideration of the EB processes and the laser's role in the acceleration of the radiative deexcitation of the nucleus, we emphasize that the above example clearly shows again the advantage of the use of intense lasers in this field:

-due to the high intensity, lasers provide the fulfillment of the resonant conditions ensured by the multiphoton absorption (emission).

-multipolar nuclear $\gamma$-transitions are converted into the emission (absorption) of electric dipole radiation. Laser radiation transfers not only the energy to the shell to eliminate the mismatch between the atomic and nuclear transition energies, but also "transfers" angular momentum to provide the dipole character of the final radiation, which leads to an enhancement of the probability of X-ray radiation by the final electron.

Turning now to the case of the resonant atomic transitions $|i\rangle \rightarrow|n\rangle \rightarrow$ $|f\rangle \neq|i\rangle$, we use the simple estimates based on the argument that the EB mechanism can be considered as a kind of IC process where the shell electron, absorbing the $\gamma$-radiation of the nucleus, undergoes a transition not to the continuous spectrum but rather to the discrete spectrum. We shall refer to this conversion mechanism as "discrete conversion". Just as lasers assist the ordinary IC process, we also can speak here about the "laser assisted discrete internal conversion". In the case of tuning by laser quanta to remove the mismatch between nuclear and atomic transition frequencies, we refer to the "resonant discrete internal conversion."

This way of treating the acceleration of the nuclear deexcitation process by resonant laser photons presents an alternative method of the theoretical study of the EB mechanism [26, 27]. It is quite useful since it provides parallels to the well developed and accepted methods used in studies of IC phenomena. 
The extension of the theory of IC (and "resonant" IC) to the discrete case introduces a new issue since the formal determination of the "discrete IC" coefficient $\alpha_{d}$ leads to a quantity with the dimension of energy, so that the physical interpretation of the IC coefficient as the ratio of conversion and radiative transition probabilities is lost. The reason for that is clear since the normalizations of the final state wave functions for the continuous and discrete states are different.

This view of the EB process as an IC process for discrete electron final state gives for the discrete conversion coefficient $\eta^{d}=\frac{W_{I C}^{d}}{W_{\gamma}^{(1)}}$ defined as the ratio of the probabilities for discrete conversion $W_{I C}^{d}$ and direct nuclear deexcitation $W_{\gamma}^{(1)}$ introduced previously, the simple expression which does not depend on the nuclear matrix element:

$$
\eta^{d}=\frac{\alpha_{d}(M(E) L) \Gamma_{n}}{2 \pi\left(\Delta^{2}+\frac{\Gamma_{n}^{2}}{4}\right)}
$$

where $\alpha_{d}$ for the $E$ type transition (in our case of the ${ }^{235} U$ nucleus) has the form:

$$
\alpha_{d}(E L) \approx \frac{\alpha L}{L+1}(2 j+1)[(2 L-1) ! ! \mathbf{M}]^{2} \omega_{I F}^{-2 L-1}
$$

and $\mathbf{M}$ is the matrix element for the atomic transition $|i\rangle \rightarrow|f\rangle$

$$
\mathbf{M}=\left\langle f\left|r^{-L-1}\right| i\right\rangle=\int_{0}^{\infty} g_{i}(r) g_{f}(r) r^{1-L} d r
$$

where $g_{i(f)}$ is the nonrelativistic (large component) radial wave function for the electron in the $|i\rangle(|f\rangle)$ state. For the ${ }^{235 m} U$ isomeric state, in the laser free case of a large resonant defect, $\Delta \gg \Gamma_{n}$, we obtain $\eta^{d} \approx \frac{\alpha_{d}(E 3) \Gamma_{n}}{2 \pi \Delta^{2}} \approx 2 \cdot 10^{12}$. Despite the fact that the EB mechanism (laser free case) is more probable than the direct radiative transition by twelve orders of magnitude, it is much smaller than the IC coefficient $\alpha_{I C}(E 3)\left(\approx 10^{19}\right.$ for $\left.{ }^{235} U\right)$.

In the case of discrete conversion in a laser field with resonant tuning, $\Delta-\hbar \omega_{L} \ll \Gamma_{n}$, one obtains for $\eta_{L}^{d}[26]$ : 


$$
\eta_{L}^{d}=\frac{2 \alpha_{d}(E 3)|\beta|^{2}}{\pi \Gamma_{n}}
$$

where $\beta$ determines the matrix element for the transition from the intermediate atomic shell $|n\rangle$ (here $5 f_{\frac{5}{2}}$ ) to the final state $|f\rangle$ (taken here as $6 d_{\frac{3}{2}}$ ) induced by the interaction of the laser field with the electron $H_{L e}=-e \vec{E} \vec{r}$ :

$$
\beta=\frac{1}{\Delta}\left\langle n\left|H_{L e}\right| f\right\rangle
$$

(In contrast to the previous case, here $|i\rangle \neq|f\rangle: 6 s_{\frac{1}{2}} \rightarrow 5 f_{\frac{5}{2}} \rightarrow 6 d_{\frac{3}{2}}$, see Fig. 12). The parameter $\beta$ can be calculated in the quasiclassical approximation and was found to be $\sim 0.3 a_{B}$ for the above $|n\rangle$ and $|f\rangle$ states. This gives $\eta_{L}^{d} \approx 7 \cdot 10^{18}$ for the moderate laser intensities $I \sim 10^{12} \frac{\mathrm{W}}{\mathrm{cm}^{2}}$, which is comparable with the IC coefficient for the outer atomic shells. This indicates a considerable reduction in the lifetime of the isomeric state ${ }^{235} U$ due to the influence of laser radiation. In reality, this reduction must be multiplied by $\Delta t \cdot \nu$, where $\Delta t$ is the duration of the laser pulse and $\nu$ is the pulse repetition rate. In practice, this factor is much less than unity.

For higher laser intensities $\left(I \gtrsim 10^{14} \frac{\mathrm{W}}{\mathrm{cm}^{2}}\right)$ the estimates are complicated by the formation of multiply charged uranium ions due to multiphoton ionization. This leads to additional (ionization) broadening of the $|n\rangle$ and $|f\rangle$ atomic levels which is comparable with the radiative width used above for $\Gamma_{n}\left(4 \cdot 10^{10} s^{-1}\right)$. At these intensities, $\eta^{d}$ is found to be $\sim 7 \cdot 10^{20}$ [26].

As claimed in [46], due to the fact that the ionization of the atom increases the localization of the electron wave function near the nucleus of the ion as compared to the case of a neutral atom (a well-known effect in the theory of IC), the matrix element $\mathbf{M}$ in (42) increased significantly. This leads to an the additional increase of $\alpha_{d}$ in (43), and, therefore, of $\eta_{L}^{d}$. Since the resulting value for $\eta_{L}^{d}$ is expected to be much greater than $\alpha_{I C}$ for the outer shells, the lifetime of the isomeric state ${ }^{235 m} U$ would be reduced by two or three orders of magnitude for laser intensities $I \gtrsim 10^{14} \frac{\mathrm{W}}{\mathrm{cm}^{2}}$.

At present, it is not clear how to extrapolate these results to higher intensities because of the difficulties associated with the theoretical estimate of the widths $\Gamma_{n}$ of the intermediate atomic states due to the copious formation of multiply charged ions by very strong laser fields. One expects that at high 
intensities, when the ionization broadening of the electronic states is higher than their radiative widths and where the broadening increases faster than linearly with $I, \eta^{d}$ will be reduced.

The estimates of this Section demonstrate that, in principle, the decay rate of the isomer ${ }^{235 m} U$ to the ground state can be controlled by the use of intense laser radiation.

Particularly, the EB process or the "resonance" discrete and continuous conversion can be used as a method of producing vacancies in the atomic shells. The coherent radiation resulting from filling of these vacancies, interesting enough itself, can be used for the detection and study of the resonant EB processes.

\subsection{Inverse Electron Bridge mechanism of nuclear ex- citation}

The discussions presented in the preceding subsection in connection with the "resonant discrete" IC providing a control mechanism for the decay rate of nuclear isomers apply also to the reverse process: a nucleus is excited by an electron transition when the laser radiation resonantly eliminates the mismatch between the electron level energy difference $\epsilon_{f i}=\left|\epsilon_{f}-\epsilon_{i}\right|$ and the nuclear level energy difference $E_{F I}=E_{F}-E_{I}$.

The situation where the external radiation (e.g., X-rays) transfers, through the excitation of the atomic shell, energy to a nucleus which is initially in its ground state is called inverse electron bridge (IEB) mechanism. If the energy of the electron transition $\epsilon_{f i}$ is close to the energy of the nuclear transition $E_{F I}$, then the resonantly enhanced excitation of a nucleus can be achieved by the absorption or emission -depending on whether $E_{F I}$ or $\epsilon_{f i}$ is larger - of a number of laser photons in addition to an X-ray photon. Again, the excitation of the electronic shell by the combined action of external radiation and resonant laser fields (first stage) can be more effective than the direct excitation of the nucleus by external radiation due to the dipole character of the interaction of the electron shell with the long wavelength radiation field. In the second stage the input energy is converted into energy of high multipolarity radiation needed for nuclear excitation. Of course, the applied laser field must be tuned to resonance to fulfill the requirement of energy conservation in this stage. The level scheme (a) and corresponding Feynman diagram (b) are presented in Fig. 13. 
The (approximate) theoretical treatment of this process is very similar to the laser-assisted nuclear deexcitation considered above. This is reflected in the use of the Hamiltonian (16), expressions of the type (1) for the Hamiltonians $H_{e L}$, and $H_{e x}$ describing the interactions of the electron with the laser field and the X-ray field, respectively, Eqs. (32), (33), and Eq.(34) for the wave function of the electron state "dressed" by the intense laser field, as well as (35), etc. All these approximations mean that the diagram in Fig. 13(b) must be replaced by the diagram in Fig. 14, where only the intermediate atomic state is dressed according to the sum of the diagrams shown in Fig. 15 and is described by the wave function (34), although, strictly speaking, both $|i\rangle$ and $|f\rangle$ states have to be considered as dressed states, too.

The dressed intermediate electronic state is able to absorb the laser photons tuned to resonance before the nuclear excitation takes place.

\subsection{Some estimates}

We omit detailed calculations which are similar to the previous one for obtaining formulae (36), (37), and (38).

Let us take again, as an example, the ${ }^{235 m} U$ isomeric state [10, 40, 55] with $E_{I F}=73.5 \mathrm{eV}, L=3$ and $O_{4}\left(5 d_{\frac{3}{2}}\right)\left(E_{B}=105 \mathrm{eV}\right)$ and $P_{3}\left(6 p_{\frac{3}{2}}\right)\left(E_{B}=32.3\right.$ $\mathrm{eV})$ shells as $|i\rangle$ and $|f\rangle$ states. The intermediate state $|n\rangle$ of binding energy $E_{B}=2.14 \mathrm{eV}$ can be excited from the initial $O_{4}$ shell by the absorption of a soft X-ray photon with energy $\hbar \omega_{x}=103 \mathrm{eV}$ if no additional laser photon is absorbed $(\mathrm{N}=0$ case). The effective charges and the principal and angular momentum quantum numbers of the electronic states are:

$|i\rangle: Z_{\text {eff }}=13.89, n_{i}=5, \ell_{i}=2\left(j_{i}=\frac{3}{2}\right),\left(E_{B}=105 \mathrm{eV}\right)$

$|n\rangle: Z_{\text {eff }}=3.173, n=8, \ell=3$ or $1,\left(E_{B}=2.14 \mathrm{eV}\right)$

$|f\rangle: Z_{\text {eff }}=9.245, n_{f}=6, \ell_{f}=1\left(j_{f}=\frac{3}{2}\right),\left(E_{B}=32.3 \mathrm{eV}\right)$.

The energy mismatch is $0.8 \mathrm{eV}$, i.e., tuning of the laser photon around $\hbar \omega_{L} \approx$ $1 \mathrm{eV}(\mathrm{N}=1)$ will result in a resonant excitation of ${ }^{235} U$ from the ground state to its isomeric state ${ }^{235 m} U$.

Using an intensity of the laser field of $I=10^{11} \frac{\mathrm{W}}{\mathrm{cm}^{2}}$ (to avoid the power broadening of the atomic widths) one obtains for $\eta_{\gamma}$ defined as the ratio of the yield for nuclear excitation by laser-assisted IEB process to the yield of direct nuclear excitation by $\gamma$-ray absorption, the value $\eta_{\gamma} \approx 3 \cdot 10^{12}$ [54.

We conclude with the statement that the combined application of an $\mathrm{X}$-ray source and an intense laser beam for nuclear excitation can be more effective than the direct $\gamma$-excitation if among the atomic electron shells there 
are two with an energy difference close to the nuclear excitation energy. The exact tuning of the electron transition to resonance is established by applying an intense laser of appropriate photon energy and intensity. Under these conditions, the laser-assisted IEB mechanism opens a realistic method for nuclear excitation with energy differences $E_{I F}$ between the excited and ground states of the order of several $\mathrm{keV}$.

Concluding this Section, we would like, for the sake of completeness, to mention that there exists an alternative way to induce the nuclear transitions by producing electron holes in the atomic shells by a laser induced plasma [56. For the example of ${ }^{235} U$ considered above, for instance, a hole is produced in the $5 d_{\frac{3}{2}}$ shell, then the electron of the above mentioned $6 p_{\frac{3}{2}}$ state transits to this hole $\left(6 p_{\frac{3}{2}}\left(\epsilon_{i}=-32.5 \mathrm{eV}\right) \rightarrow 5 d_{\frac{3}{2}}\left(\epsilon_{f}=-103.1 \mathrm{eV}\right)\right)$; the emitted photon excites the ground state of ${ }^{235} U\left(\frac{7}{2}\right)^{-}$to the isomeric state with $J^{P}=\frac{1}{2}^{+}$(73.5 eV, $T_{\frac{1}{2}}=26 \mathrm{~min}$.). The laser produced plasma has ahigh electron density $\left(\gtrsim 10^{19} \mathrm{~cm}^{-3}\right)$.

The calculations are analogous to the ones developed in this section for the study of the laser-assisted IEB mechanism as a means for exciting nuclei. However, the difference is that the photon spectrum of the self radiation of the plasma is continuous, with a Planck frequency distribution:

$$
n\left(\omega_{P l}\right)=\frac{2}{\pi} \frac{\omega_{P l}^{2}}{\exp \left(\frac{\omega_{P l}}{k T}\right)-1},
$$

where $T$ is the plasma temperature and $k$ is the Boltzmann constant.

The excitation of nuclei in a plasma via the IEB mechanism was investigated theoretically in [57.

Two cases are interesting here. If the plasma temperature $T$ is so high that complete ionization of the atomic levels that normally would participate in the IC process, takes place, then the IEB will occur through the discrete part of the spectrum. If, however, $T$ is too low to provide the complete ionization of such levels (i.e., $E_{I F} \gg k T$ ), then the IEB goes mainly through the continuous part of the spectrum. In [57], the ratios of the efficiencies for the excitation of nuclei by the plasma photons through the IEB mechanism and by thermal radiation [58] were calculated for both temperature cases.

For the example of the uranium nucleus $\left(6 p_{\frac{3}{2}} \rightarrow 5 f_{\frac{3}{2}}\right.$ transition) and in the case of the transitions through discrete atomic levels $\left(E_{I F} \ll k T\right)$, this ratio is $\approx 10^{17}$ [57. 
This effect was observed in [59] for $\mathrm{CO}_{2}\left(\lambda_{L}=10.6 \mu \mathrm{m}\right)$ and $\mathrm{Nd}(\lambda=$ $1.06 \mu \mathrm{m})$ lasers where the production of the isomers of the ${ }^{235} \mathrm{U}$ nucleus was observed by the detection of IC electrons from isomeric levels. The increase ( $\sim 10^{2}$ times) of the isomer yield obtained with the shorter wavelength laser was observed, too.

In [60, 61, 62] the capture mechanisof electrons from the plasma continuum by an outer electronic shell is predicted.

\section{Lasers in the study of anomalously low- lying isomeric nuclear states: $\left({ }^{229 m} T h\right)$}

\subsection{Introductory remarks}

In this section we use the ideas and theoretical treatments which were already discussed and applied in some detail in the previous sections. We think that the specific role of the phenomenon of anomalously low-lying nuclear state $(\approx$ several $\mathrm{eV})$ justifies a detailed discussion. The problem has a relatively long history.

In 1989 the results of the first relatively accurate experimental determination of energies and intensities of $\gamma$ transitions populating the ground state and first excited state of ${ }^{229} \mathrm{Th}$ obtained from the $\alpha$ decay of ${ }^{233} \mathrm{U}$ $\left({ }^{233} U \rightarrow{ }^{229} T h+\alpha\right)$ were reported [63]. Earlier, in the study of the rotationalband structure of ${ }^{229} \mathrm{Th}$ it was concluded [64] that the excited $\frac{3}{2}^{+}[631$ [ ] state is located quite close (within $100 \mathrm{eV}$ ) to the ${ }^{229} \mathrm{Th}$ ground state $\left(\frac{5}{2}^{+}[633]\right)$. This statement was indirectly supported by the study of the ${ }^{230} \mathrm{Th}(d, t){ }^{229} \mathrm{Th}$ reaction [65, 66]. Later it was established that the energy difference between the isomeric state and ground state of ${ }^{229} \mathrm{Th}$ does not exceed $10 \mathrm{eV}$ 67. In the paper quoted above [63 this upper limit was reduced to $5 \mathrm{eV}((-1 \pm 4)$ $\mathrm{eV})$. Unfortunately, the techniques used in these investigations did not make it possible to determine the energy of the isomeric state $E_{i s}$ more accurately or to measure its half-life time. Furthermore, it is worth mentioning that from these studies it can not definitely be excluded that the state $\frac{3}{2}^{+}[631]$ is the ground state of ${ }^{229} \mathrm{Th}$ as opposed to the state $\frac{5}{2}^{+}$[633] [27, 63].

In a recent paper [68], in an attempt to improve the value for the energy of the ${ }^{229} \mathrm{Th}$ isomeric level, the authors of [63] remeasured the energies of a

\footnotetext{
${ }^{9}$ Set $\left[N n_{Z} \Lambda\right]$ are the Nilsson model quantum numbers
} 
number of $\gamma$ rays associated with the $\gamma$-decay of ${ }^{233} U$. Compared with their earlier study, they considered more $\gamma$ rays in the ${ }^{229} \mathrm{Th}$ spectrum, used more well-measured energy calibration and reference lines, and more detectors with better energy resolution. They were able to more closely match the counting rates in the $\gamma$ ray peaks whose relative energies had to be measured, and to reduce systematic errors. More than $111 \gamma$-ray spectra were measured, and a value of $3.5 \pm 1.0 \mathrm{eV}$ was determined for the energy of the low-lying isomeric level of ${ }^{229} \mathrm{Th}$. In these undoubtedly improved measurements, again, it was assumed that the $\frac{3}{2}^{+}$state lies above the $\frac{5}{2}^{+}$state.

The importance of the existence of low-lying isomeric states, unusually low on a nuclear energy scale, is obvious. This includes not only nuclear physics itself, but also optics, solid-state physics, lasers, plasma, etc. For example, considering the great sensitivity of these levels to the electronic stucture, the lifetimes of such states are expected to depend on the chemical and physical environment in which these isomers are embedded. Thus, the knowledge of the life-time, e.g., of ${ }^{229 m} \mathrm{Th}$ in different chemical and physical environments could provide valuable information for atomic and condensed matter physics. It is important to emphasize that closely spaced levels with energy spacing of several tens $\mathrm{eV}$ are encountered quite frequently at excitation energies of the order of $\sim 10^{2} \mathrm{eV}$. However, they have vanishingly low probabilities in comparison with the background connected with decays to low-lying states or to the ground state. These transitions can be studied only in the isomer decays of low-lying levels. The frequently considered example of ${ }^{235} \mathrm{U}$ is typical from this point of view. However, its isomer, as we discussed already, decays via the IC channel, despite the fact that the efficiency of the EB (or IEB) processes is very high for ${ }^{235} U(\eta \gg 1$; see Section 5$)$ it is small on the scale of the IC process, which dominates here $\left(\alpha_{I C} \approx 10^{19}\right)$. For other examples considered above, (e.g., ${ }^{93 m} \mathrm{Nb}$ or ${ }^{193 m} \mathrm{Ir}$ ), $\eta<1$ and again IC is the dominant process. From this point of view, the situation of ${ }^{229} \mathrm{Th}$ is entirely different: the ionization potential of the thorium atom is $6.08 \mathrm{eV}$, so that a transition of the isomeric nucleus ${ }^{229 m} \mathrm{Th}$ to the ground state via IC with a final unbound electron is impossible (at least, for an isolated atom).

These considerations are the basis for the high interest in laser radiation as a means to accurately study the properties of ${ }^{229} \mathrm{Th}$ (energy level differences, lifetime of isomeric nucleus, etc.). 


\subsection{Deexcitation of isomeric state by EB mechanism}

First, we consider the deexcitation of the isomeric state of the ${ }^{229} \mathrm{Th}$ nucleus to its ground state using the generally accepted ordering of the levels shown in Fig. 16. Fig. 6 gives the Feynman diagram describing the EB process.

The dominant nuclear transition is of M1 type. The allowed E2 transition is damped by the ratio $\left(\frac{R_{A}}{\lambda_{N}}\right)^{2}$, where $\lambda_{N} \gtrsim 2.5 \cdot 10^{-5} \mathrm{~cm}$ is the wavelength of the nuclear radiation and $R_{A}$ is the nuclear radius.

In calculating the EB process probability, as before, we made a number of simplifications: we considered only the direct diagram; here, as in many other cases (see, e.g., 47]) the exchange diagram is small. Second, we used again the single-level approximation which is here well justified by the concrete analysis of the atomic level structure [52. The final goal is the calculation of the ratio $\eta$ for the probabilities $W_{\gamma}^{(3)}$ and $W_{\gamma}^{(1)}$ introduced earlier (see Section 5, expressions (36), (37), and (38) using the traditional method or (40) - (44) based on the "discrete IC" [26, 52]). In [52 the initial electronic state was taken as $|i\rangle=6 d_{\frac{3}{2}}$, with energy $\epsilon_{i}=-4.2 \mathrm{eV}$, intermediate $|n\rangle=7 p_{\frac{1}{2}}, \epsilon_{n}=$ $-2.9 \mathrm{eV}$ (values for $\epsilon_{i}, \epsilon_{n}$ are theoretically estimated in [52]).

Calculations [52 have shown that $\eta$ is less than unity, i.e., the direct nuclear deexcitation is the dominant channel, if the nuclear transition energy difference $E_{i s}=E_{I}-E_{F}$ is smaller than $\epsilon_{n}-\epsilon_{i}=1.3 \mathrm{eV}$, leading to the expected lifetime of the isomer $T_{\frac{1}{2}} \gtrsim 10 \mathrm{~d}$.

If the energy of the nuclear transition is at resonance with the energy of one of the allowed atomic transitions, $\Delta \equiv E_{I}-E_{F}-\left(\epsilon_{n}-\epsilon_{i}\right) \approx 0$ (e.g., $6 d_{\frac{3}{2}} \rightarrow 6 d_{\frac{5}{2}}$ or $7 s_{\frac{1}{2}} \rightarrow 6 d_{\frac{3}{2}}$ ), the EB probability increases sharply. Of course, ensuring the condition $\Delta=0$ demands a much better knowledge of $E_{i s}$ than is available today $\left((3.5 \pm 1) \mathrm{eV} 68\right.$ ). For the range $2 \leq E_{i s} \leq 5 \mathrm{eV}$ theoretical calculations give $T_{\frac{1}{2}} \gtrsim 10 \mathrm{~min}$ [52, whereas the authors of 68] claim that $T_{\frac{1}{2}} \gtrsim 45 \mathrm{hr}$ for $E_{i s}=3.5 \mathrm{eV}$ (M1 transition). Taking into account the $\pm 1 \mathrm{eV}$ uncertainty in the excited state energy, $T_{\frac{1}{2}}$ could be as long as $\approx$ $120 \mathrm{~h}$ or as short as $\approx 20 \mathrm{~h}$.

However, one has to realize that the half-life time of an anomalously low-lying nuclear level $\left(E_{i s} \approx\right.$ several $\left.\mathrm{eV}\right)$ is a very subtle quantity. It is influenced by the interaction not only with the electronic shell, but, due to the extremely small value of the isomeric state energy, it will be affected also by the physical and chemical properties of the sample containing the atoms of ${ }^{229} \mathrm{Th}$. Experimental and theoretical studies of such a phenomena may open new interesting directions into atomic and condensed matter physics. 
We now turn to the question of the laser-assisted deexcitation of the isomeric ${ }^{229} \mathrm{Th}$ state to its ground state using the resonant, discrete IC with bound electrons, applying a laser field with the appropriate frequency. As we saw above and in Section 5, this leads to a drastic acceleration of the nuclear isomeric decay [52, 27]. Very recently, the probability for this process was recalculated [69]. As atomic levels, in contrast of [52], the states $7 s_{\frac{1}{2}}$ and $8 s_{\frac{1}{2}}$ were considered with energies taken from [70] and [27], respectively. The energy difference of $3.713 \mathrm{eV}$ between these states is very close to the 3.5 $\mathrm{eV}$ nuclear decay energy. This electronic transition (M1 excitation) requires needs a photon energy of $0.1065 \mathrm{eV}$ in a resonant two-photon process. The experimental signal for the excitation could be the photon emission during the decay of the excited $8 s_{\frac{1}{2}}$ state via the $7 p_{\frac{1}{2}}$ and $7 p_{\frac{3}{2}}$ states.

There is another possibility [27, 52] based on the absorption of a single laser photon which excites the $8 s_{\frac{1}{2}}$ state to the $8 p_{\frac{1}{2}}$ final state (first-order process in the laser interaction whereas the first scheme corresponds to the second-order process). In this case the necessary photon energy is $0.712 \mathrm{eV}$.

Because the probability increases in the first-order case only linearly with intensity, the second-order (in the laser field) process was chosen in 669 since its probability has a quadratic increase and it is not small in absolute value. Use of a resonant laser field is crucial since in the laser free case the deexcitation of the $\frac{3}{2}^{+}$nuclear state with excitation of the electron states given above has only a very small probability $\left(\sim 5 \cdot 10^{-13}\right)$ [69].

Fig. 17 [69] shows the strong dependence of the deexcitation probability of the nuclear isomeric $\frac{3}{2}^{+}$state of ${ }^{229} T h$ for the $7 s_{\frac{3}{2}} \rightarrow 8 s_{\frac{1}{2}}$ M1 atomic transition on the laser intensity $I$. The laser photon energy is fixed to the energy of a resonant two-photon process with $E_{i s}=3.5 \mathrm{eV}$ (solid line), 4.0 $\mathrm{eV}$ (long-dashed line), and $3.0 \mathrm{eV}$ (short-dashed line) or for the resonant four-photon process for an energy of $3.5 \mathrm{eV}$ (dotted line).

We observe a strong increase with $I$, until the ionization thresholds of the electron states are reached where the probability drops strongly. As seen in Fig. 17, the absolute value of the deexcitation probability depends on the laser photon energy. One can also notice the quadratic dependence of the atomic shell excitation probability on $I$ for the two-photon process and the fourth power of the intensity increase (with smaller probability).

In the paper 669 several examples $\left({ }^{161} \mathrm{Dy},{ }^{189} \mathrm{Os},{ }^{193} \mathrm{Ir},{ }^{197} \mathrm{Au},{ }^{235} \mathrm{U}\right.$, ${ }^{237} \mathrm{~Np}$ ) are considered from the point of view of the IEB mechanism leading to the excitation of nuclei. The paper considers the laser intensity up to 
$10^{24} \frac{\mathrm{W}}{\mathrm{cm}^{2}}$. We highly recommend this paper to the interested reader.

\subsection{Pumping isomers by laser resonance}

The role of lasers is important also for the inverse process, i.e., the pumping of the ground state nuclei ${ }^{229} \mathrm{Th}$ to its isomeric state. As we stressed before, the $E_{i s}=E_{F}-E_{I}$ is known today with poor precision, which makes the direct application of the laser light to the nucleus in the ground state ineffective. The solution is to use the IEB mechanism which was discussed in Section 5 and which provides the non-radiative excitation of the nucleus in an electron transition induced by the laser radiation field. It is necessary to tune the laser light to the wavelengths of well known atomic transitions. In this case, even if there is a significant difference between the energies of the atomic $\left(\epsilon_{i n}\right)$ and nuclear $\left(E_{I F}\right)$ transitions involved, the excitation of the nucleus has a large enough probability to provide for an efficient pumping of the isomeric state, which in turn opens the possibility to measure the energy and the lifetime of the low-lying nuclear isomeric state.

The (third order in the electromagnetic interaction) process is described by the Feynman diagrams in Figs. 14 and 15. At the resonance $\left(\hbar \omega_{L}=\right.$ $\left.\epsilon_{n}-\epsilon_{i}\right)$ which provides more effective pumping, the (direct) diagram of Fig. 14 dominates and the single level approximation is reliable. These features essentially simplify the calculations.

Below, we follow the considerations given in the papers [71, 72]. For the wave functions of the initial $(i)$, intermediate $(n)$, and final $(f)$ shell electrons with energies $\epsilon_{i, n, f}$ and widths $\Gamma_{i, n, f}$ we take

$$
\psi_{i, n, f}(\vec{R}, t)=e^{-i\left(\epsilon_{i, n, f}-i \frac{\Gamma_{i, n, f}}{2}\right) t} \psi_{i, n, f}(\vec{R}),
$$

where the initial electronic shell $|i\rangle$ of the thorium atom is taken as $6 d^{2} 7 s^{2}$ with $\epsilon_{i}=-6.08 \mathrm{eV}$, and $\Gamma_{i}$ is taken to be zero.

Because the transition is of magnetic type (M1), we use the relativistic electronic wave functions $\psi(\vec{R})$ including the large $g(\vec{R})$ and small $f(\vec{R})$ component with the normalization condition

$$
\int d R \cdot R^{2}\left(g^{2}(R)+f^{2}(R)\right)=1
$$

The nuclear wave functions of the initial stationary state with energy $E_{I}$ and the final isomeric state with energy $E_{I}$ and width $\Gamma_{i s}$ are 


$$
\begin{aligned}
& \psi_{I}(\vec{r}, t)=e^{-i E_{I} t} \psi_{I}(\vec{r}) \\
& \psi_{F}(\vec{r}, t)=e^{-i\left(E_{F}-i \frac{\Gamma_{i s}}{2}\right) t} \psi_{F}(\vec{r}) .
\end{aligned}
$$

A matrix element of the 3rd order process described by the direct diagram of Fig. 14 includes, in addition to the wave functions for the electrons and the nucleus, the electromagnetic interactions of the photon with the electrons, $j^{\mu}(\vec{R}) A_{\mu}(\vec{R})$, and nucleus $J^{\mu}(\vec{r}) A_{\mu}(\vec{r})$, respectively, where

$$
j_{i f}^{\mu}(\vec{R})=e \bar{\psi}_{f}(\vec{R}) \gamma^{\mu} \psi_{i}(\vec{R})
$$

is the electron electromagnetic current and

$$
J_{I F}^{\mu}(\vec{r})=e \psi_{F}^{+}(\vec{r}) \hat{J}^{\mu} \psi_{I}(\vec{r})
$$

where $\hat{J}^{\mu}$ is the four-vector of the nuclear electromagnetic current not specified here. Furthermore, the matrix element includes the photon, $D_{\mu \nu}$, and the intermediate electron propagators.

The standard calculations include the expansion of the photon vector potentials and propagator in terms of multipoles (see, e.g., [73, 74]). Furthermore, the matrix element of the nuclear current (49) is expressed in terms of the reduced matrix elements [29], taking into account the selection rules for the nuclear transition, etc. Finally, one obtains the following expression for the cross section $\sigma^{(3)}$ of the IEB process $[72$ at resonance with the atomic $|i\rangle \rightarrow|n\rangle$ transition $\left(\hbar \omega_{L}=\epsilon_{n}-\epsilon_{i}\right)\left(\Gamma_{i}=0, \Gamma_{i s} \ll \Gamma_{n}\right)$ :

$$
\begin{aligned}
\left.\sigma^{(3)}=\frac{\lambda_{L}^{2}}{\pi}\left(1+\frac{\Gamma_{f}}{2 \Gamma_{n}}\right) \frac{\Gamma_{A}^{r}\left(\omega_{L} ; i\right.}{\rightarrow}, n, M(E) 1\right) & \Gamma_{n} \\
& \cdot \frac{E_{\text {int }}^{2}\left(E_{F}-E_{I} ; M 1 ; n \rightarrow f, I \rightarrow F\right)}{\left[\epsilon_{n}-\epsilon_{f}-\left(E_{F}-E_{I}\right)\right]^{2}+\Gamma_{n}^{2}\left(1+\frac{\Gamma_{f}}{2 \Gamma_{n}}\right)^{2}}
\end{aligned}
$$

This expression is valid when the characteristic width of the laser line is comparable in magnitude with the atomic intermediate state width $\Gamma_{n}$ : $\Delta \omega_{L} \approx \Gamma_{n}$. Here, $\Gamma_{A}^{r}$ is the width of the atomic radiative transition $i \rightarrow n$ 
of multipolarity $M(E) I$, and $E_{i n t}^{2}$ is the (energy) ${ }^{2}$ of the interaction of the electron transition current $j_{n f}^{\mu}(\vec{R})(48)$ and the nuclear current $J_{I F}^{\mu}(\vec{r})(49)$ averaged over the initial and summed over the final states:

$$
E_{\text {int }}^{2}=\sum_{\substack{I F \\ n f}}\left|\int d^{3} r d^{3} R j_{n f}^{\mu}(R) D_{\mu \nu}(\omega ; \vec{R}-\vec{r}) \cdot J_{I F}^{\nu}(\vec{R})\right|^{2},
$$

where $D_{\mu \nu}(\omega ; \vec{R}-\vec{r})$ is the Greens function of the photon in the coordinatefrequency representation 75

$$
D_{\mu \nu}(\omega ; \vec{R}-\vec{r})=\frac{-g_{\mu \nu} \exp [i \omega|\vec{R}-\vec{r}|]}{|\vec{R}-\vec{r}|} .
$$

With $\left|\omega_{I F} \cdot R\right| \ll 1$, (51) is reduced to the form

$$
E_{\text {int }}^{2}\left(\omega_{F I} ; M 1\right)=\frac{1}{4} \Gamma_{A}^{r}\left(\omega_{I F} ; n \rightarrow f ; M 1\right) \cdot \Gamma_{N}^{r}\left(\omega_{I F} ; I \rightarrow F, M 1\right)\left(1+\delta^{2}\right),
$$

where $\Gamma_{N}^{r}$ is the probability for the radiative nuclear transition and

$$
\delta=\frac{\operatorname{Im}\left[\mathbf{M}_{M 1}\left(\omega_{I F}\right)\right]}{\operatorname{Re}\left[\mathbf{M}_{M 1}\left(\omega_{I F}\right)\right]}
$$

is the analog of the corresponding well-known quantity in the theory of IC, and

$$
\mathbf{M}_{M 1}(\omega)=\left(\varkappa_{n}+\varkappa_{f}\right) \int_{0}^{\infty} d r \cdot r^{2} h_{1}^{(1)}(\omega r)\left[g_{n}(r) f_{f}(r)+f_{n}(r) g_{f}(r)\right],
$$

where $h_{1}^{(1)}(x)$ is the spherical Hankel function and $\varkappa=(\ell-j)(2 j+1)$, and $\ell$ and $j$ are the orbital and total angular momentum of the electron in the corresponding shell. The matrix element $\mathbf{M}_{M 1}(\omega)$ is calculated numerically in [71, 72]. Typical values for the important quantity $E_{\text {int }}^{2}$ vary for different atoms in the range $\left(10^{-10}-10^{-12}\right) \mathrm{eV}^{2}$. In the already mentioned recent paper 66] this quantity was calculated for several nuclei on the basis of the 
adiabatic description of the dressed electronic states in the laser field. The values obtained there for $E_{\text {int }}^{2}$ coincide within one order of magnitude with the results of [71, 72]. However, in the case of the popular ${ }^{235} U$ the result is in drastic disagreement with the calculation of [71, 72].

In the situations where the width $\Delta \omega_{L}$ of the laser is larger than the widths of the atomic states $\Gamma_{n, f}$, it is necessary to carry out the integration of (50) obtained for monochromatic laser photons over the shape of the laser line $g\left(\omega_{L}-\omega_{0}\right)$ with $\int g\left(\omega_{L}-\omega_{0}\right) d \omega_{L}=1$.

At the resonance, $\omega_{0}=\omega_{i n}$, with the conditions $\Gamma_{n, f} \ll \hbar \Delta \omega_{L}$, and $\Gamma_{f}, \Gamma_{i s} \ll \Gamma_{n} \ll \epsilon_{n}-\epsilon_{f}-E_{F I}$, one has:

$$
\sigma_{\text {res }}^{(3)} \approx \lambda_{L}^{2} \frac{\Gamma_{A}^{r}(i \rightarrow n ; M(E) 1) E_{\text {int }}^{2}\left(\omega_{I F} ; M 1 ; n \rightarrow f, I \rightarrow F\right)}{\left[\epsilon_{n}-\epsilon_{f}-\left(E_{F}-E_{I}\right)\right]^{2}} \cdot\left\{\begin{array}{ll}
\frac{1}{\pi} & \text { for } L \\
\left(\frac{\ln 2}{\pi}\right)^{\frac{1}{2}} & \text { for } G(56)
\end{array},\right.
$$

where L stands for Lorentzian and $\mathrm{G}$ for Gaussian line shapes.

This expression, along with (50), (53), and (55), is the basis for the estimates below.

\subsection{Pumping efficiency}

The arguments given in the previous subsection showed that the stimulation of the pumping of the isomeric state ${ }^{229 m} T h$ by the IEB mechanism requires a careful study of the structure and properties of the atomic levels of the thorium atom. However, information about the ${ }^{229} \mathrm{Th}$ atom is not complete. In [76 only the energies of the levels are given, while in [77 energies, spins, and parities for some of the states are presented.

Two cases are interesting in the IEB mechanism for the thorium atom 71, 72,

i) The atomic transition $|i\rangle \rightarrow|n\rangle$ has the multipolarity $M 1$ (i.e., the same as the isomeric nuclear transition $|I\rangle \rightarrow|F\rangle$ ) and the final state of the electron shell, $|f\rangle$, coincides with the initial $|i\rangle$ one ("elastic" IEB). The essential parameter $\Gamma_{A}^{r}$, defining the cross section $\sigma^{(3)}$ of the IEB mechanism (see(50)) can be under these conditions $\sim\left(10^{-1}-10^{-2}\right) \Gamma_{n}$. This situation is realized in the case, for example, $|n\rangle=6 d^{3} 7 s\left({ }^{5} F_{3}\right)$, with $\epsilon_{n}=-5.15 \mathrm{eV}$ [78].

ii) The $|i\rangle \rightarrow|n\rangle$ transition is of $E 1$ or $M 1$ type but the final state does not coincide with initial one ("inelastic" IEB mechanism). One possible realization is $|n\rangle=6 d 7 s^{2} 7 p\left({ }^{3} F_{2}\right), \epsilon_{n}=-4.74 \mathrm{eV}$ and $|f\rangle=5 f 6 d 7 s^{2}\left({ }^{3} F_{2}\right)$, 
$\epsilon_{f}=-5.06 \mathrm{eV}$ [78. Under favorable conditions, for an atomic $E 1$ transition $|i\rangle \rightarrow|n\rangle$ and small mismatch between $E_{F}-E_{I}$ and the energy of one of the atomic transitions $|n\rangle \rightarrow|f\rangle(\approx 0.1 \mathrm{eV})$ the excitation cross-section due to the IEB mechanism may reach a value of the order $\sigma_{\text {res }}^{(3)} \approx 10^{-20}-10^{-21} \mathrm{~cm}^{2}$ for ordinary lasers with $\frac{\Delta \omega_{L}}{\omega_{L}} \approx 10^{-6}$. At less favorable conditions, i.e., fixing the value for the mismatch $\tilde{\Delta}=E_{F I}-\epsilon_{n f}$ between the atomic and nuclear transitions in the energy denominators of Eqs. (50), (56) for $1 \mathrm{eV}$, for $\omega_{L}=$ $1-5 \mathrm{eV}$ and $\frac{\Delta \omega_{L}}{\omega_{L}} \approx 10^{-6}$, and taking for $E_{\text {int }}^{2}$ values between $10^{-10}$ and $10^{-12}$ it was found that $\sigma^{(3)} \approx 10^{-23}-10^{-25} \mathrm{~cm}^{2}$.

By moving "up" in the energies of the excited atomic states, one can find suitable atomic levels which should be stimulated in the case $E_{i s}>2 \mathrm{eV}$. Notice that the value for $\sigma^{(3)}$ found is a lower estimate. Due to the relatively high density of the excited atomic levels of ${ }^{229} \mathrm{Th}$, the actual value of $\tilde{\Delta}$ may be much less than $1 \mathrm{eV}$.

For the elastic or inelastic $M 1$ atomic transitions $|i\rangle \rightarrow|n\rangle$, the cross section $\sigma^{(3)} \approx 10^{-25}-10^{-26} \mathrm{~cm}^{2}$ due to the small value of $\Gamma_{A}^{r}(M 1)\left(\approx \alpha^{2} \Gamma_{A}^{r}(E 1)\right)$.

Defining the excitation efficiency $\xi$ as the ratio of the number of produced isomeric nuclei ${ }^{229 m} \mathrm{Th}$ to the number of thorium atoms exposed to the laser light, we can write:

$$
\xi=\sigma^{(3)} \tau \phi_{L}
$$

where $\tau$ is the irradiation time and $\phi_{L}$ is the flux density of the laser photons, $\phi_{L}=\frac{I}{\hbar \omega_{L}}=\rho_{\gamma} c$, and $\rho_{\gamma}$ is the laser photon density. To lower the background from the natural $\alpha$-decay of the thorium nuclei with the activity $A_{T h}(\alpha)=3.2 \cdot 10^{-12} N(T h)$ decays per second, where $N(T h)$ is the number of thorium nuclei, let us estimate the induced activity for a very small amount of thorium atoms taking a sample of mass $10^{-8} \mathrm{~g}$ evaporated as a layer of thickness $10 \AA\left(N(T h)=10^{13}\right)$ onto a backing of diameter $1 \mathrm{~mm}$. Under these conditions a fairly small absorption of the photons emitted in the decay of the ${ }^{229 m}$ Th isomers in the sample is guaranteed.

Irradiation of this target with a laser beam of power $100-200 \mathrm{~mW}$ focused onto $\approx 1 \mathrm{~mm}$ for the $\tau=10^{2}-10^{3}$ s gives the efficiency $\xi \approx 0.01-1$ with $\sigma^{(3)}=10^{-23}-10^{-24} \mathrm{~cm}^{2}$. As a result, the $\gamma$-activity of the isomer ${ }^{229 m} \mathrm{Th}$ will be $10^{5}-10^{7} \mathrm{~Bq}$ (for the $T_{\frac{1}{2}}^{i s} \approx 10 \mathrm{~d}$ ). Thus, using a tunable laser one can stimulate a suitable atomic state with energy greater than the energy of 
the isomeric transition $E_{F}-E_{I}$, and the nuclear excitation will take place via the decay channels of this atomic state.

In conclusion, the example of the ${ }^{229} \mathrm{Th}$ nuclei shows that the optical laser pumping of low-lying isomeric nuclear states via the IEB mechanism is a realistic and relatively simple tool to measure and study lifetimes and energy levels.

\subsection{Modified IEB Mechanism in the study of ${ }^{229 m} \mathrm{Th}$}

There exists a modification to the use of the IEB mechanism for the excitation and study of the properties of the isomeric state of the thorium nucleus [79]. Below, we follow the paper [79]. It is connected with the study of the photon energy which emerges during the nuclear excitation. Schematically, it looks as follows (Fig. 18): A laser beam excites the atomic electron to a state of angular momentum defined by the selection rules in the subsequent deexcitation of the shell. The electronic deexcitation leads to the excitation of the nucleus and, simultaneously, to the photon emission.

In the following, we assume that the electron has already reached the excited state $|n\rangle$. The nuclear quadrapole transition $\frac{5}{2}^{+} \rightarrow \frac{3}{2}^{+}\left(\right.$or $\frac{3}{2}^{+} \rightarrow \frac{5}{2}^{+}$, if the ground nuclear state of ${ }^{229} \mathrm{Th}$ has angular momentum $\frac{3}{2}^{+}$and not $\frac{5}{2}^{+}$ as it is usually claimed), with photon emission in the dipole regime, imposes the condition $j_{i}+j_{n} \geq 3$.

Thus, a new line appears in the optical spectrum of the laser excited ${ }^{229} \mathrm{Th}$ spectrum, its frequency is defined by the relation

$$
\hbar \omega=\hbar \omega_{n i}-\hbar \omega_{F I}
$$

That opens the possibility of the

i) precise determination of the energy of the ${ }^{229 m} \mathrm{Th}$ isomeric nucleus by measuring the photon frequency $\omega$;

ii) solution of the uncertainty concerning the question of the angular momenta of the nuclear ground and isomeric states $\left(\frac{5}{2}^{+}\left(\right.\right.$or $\left.\frac{3}{2}^{+}\right)$and $\frac{3}{2}^{+}\left(\right.$or $\left.\frac{5}{2}^{+}\right)$, corresponding to the sign of the difference $\left.\omega_{F}-\omega_{I} \lessgtr 0\right)$. Furthermore, once the magnitude of the emitted photon frequency $\omega$ is known, the process can be accelerated by the resonant application of the laser with frequency $\omega_{L}=\omega$. The simplified calculations are very similar to the calculations of Sections 4 and 5 for the IC and EB processes. The role of the initial electronic state $|i\rangle$ 
plays here the laser-excited, dressed electronic state $|n\rangle$. Again, emission of the photon frequency $\omega$ is treated quantum-mechanically.

The nuclear transition is a quadruple type, $\mathrm{L}=2$. The initial, excited atomic state is taken as:

$$
i:|n\rangle=8 p 7 s^{2}, n_{i}=8, l_{i}=1 .
$$

We take the final electronic state $|f\rangle$ as

$$
f:|f\rangle=6 d^{2} 7 s^{2}
$$

with $n_{f}=6, l_{f}=2$ satisfying the above mentioned condition about the angular momenta involved.

Since the initial electronic state in our scheme is the excited state, we have to take into account the energy distribution of this state around its central energy $\epsilon_{i 0}$ with width $\gamma_{i}$ (line shape)

$$
\rho\left(\epsilon_{i}\right)=\frac{\gamma_{i}}{2 \pi} \frac{1}{\left(\epsilon_{i}-\epsilon_{i 0}\right)^{2}+\frac{\gamma_{i}^{2}}{4}}
$$

as a weight factor in the integration (averaging) over the initial electronic state. In the resonance case we have $\epsilon_{i 0}=\epsilon_{f}+E_{F}-E_{I}+\hbar \omega_{L}$. The integration gives a factor $\frac{2}{\pi} \gamma_{i}^{-1}$. The laser induced IEB process at $\omega_{L}=\omega$ can be written as

$$
W_{f i}^{L}=\frac{1}{2 \pi} W_{f i} \phi \lambda_{L}^{2} \frac{1}{\gamma_{i}}
$$

where $\phi$ is the laser flux $\frac{N c}{V}\left(=\frac{I}{\hbar \omega_{L}}\right)\left(V\right.$-volume), and $W_{f i}$ is the transition probability (per unit time) of the spontaneous photon emission due to the IEB:

$$
W_{f i}=C W_{f i}^{\circ}\left|\left\langle F|| \hat{Q}_{L}|| I\right\rangle\right|^{2}\left|I_{L, n_{f} l_{f}}^{n_{i} l_{i}}\right|^{2},
$$

where

$$
W_{f i}^{\circ}=\left(\frac{r_{0}}{a_{B}}\right)^{2 L} \frac{e^{6}}{m^{2} c^{3} a_{B}^{4}} \frac{8 \pi^{2}(L+1)}{(2 L+1)\left(2 l_{i}+1\right)} \frac{C_{1}(L)}{\hbar \omega}
$$


and $\left|\left\langle F|| \hat{Q}_{L}|| I\right\rangle\right|^{2}$ is the squared reduced matrix element of the multipole moment of order $L$ between the nuclear states $|I\rangle$ and $|F\rangle$, and is expected to have a value between $10^{-2}$ and $10^{2}$ (see, 80]).

In (61), $I_{L, n_{f} l_{f}}^{n_{i} l_{i}}=a_{B}^{L+2} \int r^{1-L} R_{n_{i} l_{i}}\left(Z_{e f f}^{i}, r\right) R_{n_{f} l_{f}}\left(Z_{e f f}^{f}, r\right) d r$ (cf. (37)) which for our case $\left(n_{i}=8, l_{i}=1, L=2, n_{f}=6, l_{f}=2, Z_{\text {eff }}^{i}=2.17, Z_{\text {eff }}^{f}=4.65\right)$ gives $\left|I_{2,62}^{81}\right|^{2}=4.49 \cdot 10^{-4}$. C,$C_{1}(L)$ are constants expressed in terms of $3 j$-symbols.

For $W_{f i}^{\circ}$ we obtain:

$$
W_{f i}^{\circ}=8.7 \cdot 10^{-5} \frac{1}{\hbar \omega}, \text { in } s^{-1}
$$

and

$$
W_{f i}=1.7 \cdot 10^{-8}\left|\left\langle F|| \hat{Q}_{L}|| I\right\rangle\right|^{2} \frac{1}{\hbar \omega}, \text { in } \quad s^{-1} .
$$

The ground state of ${ }^{229} T h$ has a half-life time $T_{\frac{1}{2}} \approx 2.3 \cdot 10^{11} s$ [40. A sample of $\mathrm{N}^{229} \mathrm{Th}$ atoms has a radioactivity $A=8.1 \cdot 10^{-23} \mathrm{~N}$ (in units of $\mathrm{Ci}$ ). Thus, for a sample with $N=10^{18}$ atoms we have $A=8.1 \cdot 10^{-5}$ $\mathrm{Ci}$. If we suppose that $1 \%$ of the atoms can be populated in the desired electronic initial state by the laser field, then the photon emission rate $R$ (in $s^{-1}$ ) induced by the IEB process is

$$
R=W_{f i} \cdot 10^{16}=1.7 \cdot 10^{8}\left|\left\langle F|| \hat{Q}_{L}|| I\right\rangle\right|^{2} \frac{1}{\hbar \omega}
$$

which is larger than $10^{6} s^{-1}$. It is a lower bound since one can expect that the state $|F\rangle$ has a much smaller half-life time than the state $|I\rangle$, thus the activity of the sample will be much larger.

If we compare these estimates with estimates discussed at the end of Subsection 6.4 for a somewhat different process, where $N$ was $10^{13}$ and the obtained activity was $10^{5}-10^{7} \mathrm{~Bq}$ for an irradiation time $\sim 10^{3} \mathrm{~s}$, we obtain here an induced rate $R \sim 10^{8}-10^{10} s^{-1}$ or, for $N=10^{18}$, as used above, $R \approx 10^{13}-10^{15} s^{-1}$.

These numbers look encouraging and show that the laser-driven IEB mechanism can offer a reliable method for determining accurately the energy difference and other properties such as lifetime and angular momentum of ground and isomeric states, etc., and controlling a radioactive decay rate. 
One can go further and use the small energy difference of the $\frac{3}{2}^{+}$and $\frac{5}{2}^{+}$ levels of the ${ }^{229} \mathrm{Th}$ nucleus to modify the $\alpha$-decay rate of the nucleus in a laser driven resonant process where these two nuclear levels are mixed by the magnetic field of the laser field [4]. As a result, the $\alpha$-decay rate of the ${ }^{229} T h$ nucleus to one of the levels of the daughter nucleus can be written at the resonance as

$$
R=R_{\frac{5}{2}}+\nu R_{\frac{3}{2}}
$$

where $R_{\frac{5}{2}\left(\frac{3}{2}\right)}$ is the $\alpha$-decay rate for the $\frac{5}{2}\left(\frac{3}{2}\right)$ state in the laser free case,

$$
\nu=\frac{\pi^{2} e^{2} I}{M^{2} c^{3} \Delta \omega_{L}} \beta \tau
$$

where $M$ is the nucleon mass, $\Delta \omega_{L}$ is the band width of the laser, $\tau$ is the irradiation time and $\beta$ is the reduced M1 transition matrix element in units of the Bohr magneton. Here. $\beta$ is of order $10^{-4}$ (see, e.g. [74]). Assuming that the lifetime of the ${ }^{229 m} T h\left(\frac{3}{2}^{+}\right)$is much shorter than that of the ground state $\left(\frac{5}{2}^{+}\right)$and taking $\Delta \omega \approx 5.7 \cdot 10^{6} \mathrm{~s}^{-1}, \beta \sim 10^{-6}, \tau=1 \mathrm{~h}, I=10^{3} \frac{\mathrm{W}}{\mathrm{cm}^{2}}$, one obtains $\nu=0.19$ [4]. This last example laser assisted EB and IEB mechanisms for modifying and controlling nuclear radioactivity by the influence of light - a topic which goes back to Einstein, as shown in the epigraph to the present paper.

\section{Summary and conclusions}

In the present paper we have tried to review several applications of optical and UV-lasers for studying low-energy properties of nuclei.

The most effective tool for these applications is the use of the electronic shells of atoms as mediators between the laser field of appropriate frequency and the nuclei of interest.

All effects considered above are united by the realization of two unique advantages of lasers (besides other important properties like monochromaticity,

\footnotetext{
${ }^{10}$ Such kind of effects, of course, necessitate further detailed and accurate investigation. Particularly, in the considered example the screening of the magnetic field by atomic shells seems essential for the level mixing.
} 
polarization, etc., shared with other sources of electromagnetic radiation): i) High intensity of the laser beam makes the multiphoton absorption (emission) by atomic shells possible, thus providing the effective elimination of the mismatch between the energy differences of atomic and nuclear transitions and, furthermore, leading to resonantly enhanced effects. This possibility of using multiples of the laser frequency $\omega_{L}$ to provide the transition energy is the leading principle for the use of intense laser beams for the study of low-energy nuclear processes and properties;

ii) The dipole character of the interaction of the laser radiation with the atomic shells provides a reduction of the (usually high) multipolarity of the nuclear electromagnetic radiation, thus effectively enhancing the transition probability (the laser light transfers not only energy, but also angular momentum).

It was shown that, for intense laser beam $\left(I>10^{19} \frac{\mathrm{W}}{\mathrm{cm}^{2}}\right)$, the so called "anti-Stokes" transition, although not so probable as it was expected before, provides the effective mechanism for the deexcitation of nuclear levels located close enough to the nuclear ground state. This mechanism can be used to study specific nuclear levels and their transitions unaccessible otherwise. It was shown that the laser-assisted (induced) internal conversion (IC) is effective only for the nuclei where IC is forbidden by energy conservation in the laser-free cases. Close and below the threshold for IC the role of optical and UV-lasers is very important and leads, in principle, to observable laser induced IC coefficients. Unfortunately, for very intense lasers the hindering effects of pondermotive forces have not been taken into account yet. Thus, the theoretical study of the IC processes at very high $I$ is important. Generally, this case demands the elimination of several simplifications that we used for the study of this process. Here, time-consuming numerical calculations are required.

We have studied the role of the EB and IEB mechanisms in processes of resonant deexcitation and excitation of isomeric nuclei with emphasis on measuring their energies and lifetimes. Special attention was paid to the study of the low-lying isomeric states of the nucleus ${ }^{229 m} \mathrm{Th}$ where the use of optical and UV-lasers seems the most promising.

The existence of anomalously low-lying excited nuclear levels (as ${ }^{229 m} T h$ ) opens the real possibility to control and modify nuclear processes by optical and UV-lasers. Furthermore, the great sensitivity of these low-lying nuclear levels to the physical and chemical environment could provide valuable information for atomic and condensed matter physics, optics, plasma physics, 
lasers, etc. Almost all processes considered here were treated using many simplified approximations, thus one cannot expect to achieve precise quantitative conclusions. The present values for the transition probabilities of many processes are rather uncertain and may change considerably if more exact calculations of the electronic transition matrix elements become available in the future.

Of particular concern is the assumption of independent one-electron levels used in all cases above, which limits the predictive power of the calculations considerably. Due to many body effects, any perturbation of neighboring electronic shells by the intense laser radiation will have an influence on the binding energy and wave function of each electron. However, we believe that the essential aspects of the laser-assisted and laser-induced nuclear processes studied here will turn out to be correct and will provide useful directions for future studies.

¿From the experimental point of view, it is necessary to search for the most favorable low multipolarity transitions between inner electron states to achieve the strong nucleus-electron shell coupling.

In present (and future) applications, especially when the fulfillment of the resonance conditions by laser fine tuning becomes experimentally feasible, the knowledge of the precise energies of the nuclear and atomic states involved is of fundamental importance.

\section{Acknowledgments}

It is a pleasure to thank Werner Tornow, who attracted the author's attention to the subject, for discussions, support, and interest in the present work. I am grateful to Vladimir Litvinenko for explanations and discussions of the operation, present status and future of the Duke University FEL Facility, to Berndt Müller for discussion of theoretical topics, interest and encouragement, and to Karl Straub, who attracted my attention to the role of laser radiation in the orbital electron capture process by nuclei, and for his general interest in the present work. I also thank Holly Pulis for helping to prepare this manuscript. This work was supported in part by the US Department of Energy, Office of High Energy and Nuclear Physics, under grant No. DEFG05-91ER40619. 


\section{Appendix A: Parameters characterizing the interaction of the laser radiation with electron (dimensional arguments)}

Here we use dimensional arguments to show the role of the most important parameter of the intense laser field, the intensity, in interaction processes with electrons [81.

The intensity $I=\hbar \omega_{L} \rho c$ ( $\rho$ is the number density of laser photons) must, of course, play the essential, nontrivial role in such physical quantities as transition probability, cross section, etc. However, its inclusion should not change the dimensionality of the expressions for such quantities. This means that the density $\rho$ must always enter in the combination $\rho L^{3}$ where $L$ is some length characteristic to the effect under consideration. There are several lengths (for free electron and monochromatic radiation):

-classical radius of the electron

$$
r_{0}=\frac{e^{2}}{m c^{2}}=2.8 \cdot 10^{-13} \mathrm{~cm}
$$

-wavelength of the laser radiation

$$
\lambda_{L}\left(\approx 10^{-4}-10^{-5} \mathrm{~cm}\right), \text { or } \lambda_{L} \equiv \frac{\lambda_{L}}{2 \pi}
$$

-(reduced) electron Compton wavelength

$$
\lambda_{c}=\frac{\hbar}{m c}=3.86 \cdot 10^{-11} \mathrm{~cm} .
$$

The first two of them are classical quantities, $\lambda_{c}$ is of quantum nature. Following [81], it is illuminating to separate the most interesting dimensionless combinations of $\rho$ (which is a quantum quantity since it measures the

numbers of photons $\left(\rho=\frac{I}{\hbar \omega_{L} c}\right)$ ) and these three lengths into classical and quantum parameters:

We have (retaining for clarity $\hbar$ and $\mathrm{c}$ ):

Classical dimensionless parameters: 


$$
\begin{array}{ll}
C_{1}: & \rho \lambda_{c} r_{0}^{2}=\frac{I r_{0}^{2}}{\omega_{L} m c^{2}} \\
C_{2}: & \rho \lambda_{c} r_{0} \lambda_{L}=\frac{I r_{0} \lambda_{L}^{2}}{m c^{3}}=10^{-10} \lambda_{L}^{2} I \\
C_{3}: & \rho \lambda_{c} \lambda_{L}^{2}=\frac{I \lambda_{L}^{2}}{\omega_{L} m c^{2}}
\end{array}
$$

Quantum dimensionless parameters:

$$
\begin{array}{ll}
Q_{1}: & \rho \star_{L}^{3}=\frac{I \lambda_{L}^{3}}{\hbar \omega_{L} c} \\
Q_{2}: & \rho \star_{L}^{2} r_{0}=\frac{I \lambda_{L}^{2} r_{o}}{c} \frac{1}{\hbar \omega_{L}}
\end{array}
$$

The parameter $C_{1}$ is extremely small. The parameter $C_{2}$ has an interesting physical meaning, defining the ratio of the electromagnetic energy $r_{0} \lambda^{2} \frac{I}{c}$ of the wave with volume $r_{0} \lambda^{2}$ passing by (in "contact" with) the electron of size $r_{0}$, to the electron rest energy. We see that the relativistic effects described by $C_{2}=10^{-10} \lambda_{L}^{2} I$ are small for the $\lambda_{L}$ of interest here $\left(\lambda_{L} \sim 10^{-4}-10^{-5} \mathrm{~cm}\right)$ unless $I>10^{10} \frac{\mathrm{W}}{\lambda^{2}} \approx 10^{20} \frac{\mathrm{W}}{\mathrm{cm}^{2}}$.

The parameter $C_{3}$ has the same meaning as $C_{2}$ but with respect to a much larger volume $\lambda^{3}$. It seems to play no role since it is difficult to imagine an electron to be probed in the interaction region as large as $\lambda^{3}$. The interesting quantum parameter is $Q_{2}$ which measures the ratio of the electromagnetic energy $\frac{r_{0} \lambda^{2} I}{c}$ to the photon energy $\hbar \omega_{L}$, i.e., defines the number of the laser photons in the interaction region $r_{0} \lambda^{2}$. In other words, just this parameter describes the nonlinear, multiphoton interactions with free electron. We note that these kinds of effects were important in several processes described in the text, the only difference is that such multiphoton processes referred to bound electrons. The parameter $Q_{1}$ has the same meaning as $Q_{2}$, again with respect to the larger volume $\lambda^{3}$.

The above arguments were applied to the interaction of laser radiation with free electrons.

In some sense, the dimensional arguments are useful for atomic systems even under the influence of very intense lasers with an electric field strength $E_{0}$ of the order of $(1-5) \frac{e}{a_{B}^{2}}\left(\right.$ i.e., $\left.I=\left(7 \cdot 10^{16}-2 \cdot 10^{18}\right) \frac{\mathrm{W}}{\mathrm{cm}^{2}}\right)$. With laser 
photon energy $\hbar \omega_{L}=5 \mathrm{eV}$ the amplitude of the motion of the electrons is larger than $\frac{e E_{0}}{m \omega_{L}^{2}} \sim 30 a_{B}$.

These arguments were the basis for the authors of [82, 22] to construct an approximate theory of nuclear excitations due to the motion of the shell electrons in the very intense field of the optical and UV lasers when a laser driven highly localized current of collectively oscillating electrons induces the nuclear excitation. Each electron in this current moves on its own classical trajectories (see also 83, 84, 85]). Such a model gives the upper limit for the effect of nuclear excitations 82.

In conclusion, it is worthwhile to remark that for the atomic electrons there are additional characteristic lengths such as the Bohr radius $a_{B}$ or $\frac{a_{B}}{Z}$, so one could expect that the corresponding important parameters $C_{2}$ and $Q_{2}$ will be much larger (see also Section 4 which is devoted to the laser assisted and induced IC processes). Thus, the interaction of laser radiation of optical and UV range with a simple atomic systems would offer a very promising way to study the nonlinear effects intrinsic to the intense laser field. This issue was illustrated in the text from the point of view of low-energy nuclear physics.

\section{Appendix B: Two representations of the inter- action of radiation with matter}

As it was pointed out in the Introduction (footnote 1), the question of relating the dipole form (1) as the Hamiltonian of the matter-radiation field interaction to the general gauge invariant (non-relativistic) Hamiltonian $H=\frac{1}{2 m}(\hat{\vec{p}}-e \vec{A})^{2}(c=1)$ which gives the interaction term

$$
-\frac{e}{m} \overrightarrow{\hat{p}} \cdot \vec{A}+\frac{e^{2}}{2 m} \vec{A}^{2}
$$

has a long and controversial history (see [6]). At first glance, the problem is simply solved by applying to the wave function $\psi$ in the Shrödinger equation $\frac{i \partial \psi}{\partial t}=H \psi$ the unitary transformation

$$
\psi(\vec{r}, t)=\exp \{i \vec{A}(t) \cdot \vec{r}\} \phi(\vec{r}, t)
$$


(in the dipole approximation the vector potential $\vec{A}$ does not depend on $\vec{r})$. We obtain for $\phi(\vec{r}, t)$ the equation

$$
i \frac{\partial \phi}{\partial t}=\left[\frac{1}{2 m} \hat{p}^{2}+\vec{r} \cdot \overrightarrow{\dot{A}}(t)\right] \phi
$$

which leads, using $\vec{E}=-\overrightarrow{\dot{A}}(t)$, to the Göppert-Mayer form (1). Obviously, if $\vec{A}$ depends on $\vec{r}$, i.e., we avoid the dipole approximation, this correspondence is not valid. This equivalence is well known. But this is not the whole story. First of all, one needs to be careful if in actual studies (practically unavoidable) approximations (besides the dipole one) have to be made. Eqs. (B3) and (1) are equivalent in the dipole approximation if the wave functions $\psi$ and $\phi$ are exact solutions of the corresponding Schrödinger equations. If approximations are permitted, the equivalence may be destroyed. This situation leads, for example, to the conclusion [86] that when studying model systems with a finite number of levels, one must choose the dipole interaction in the form (1), rather than in the form (B3).

However, even if the $\psi$ and $\phi$ are the exact solutions of the Schrödinger equation, one needs to be cautious when the problem includes damping which, in the case, e.g., of the two-level system (described by a $2 \times 2$ matrix) has off-diagonal elements for the $\vec{p} \cdot \vec{A}$ interaction. It makes the dipole form $-e \vec{r} \cdot \vec{E}$ more convenient, although, if one takes into account the proper change of the damping matrix under the transformation (B2), they are equivalent.

Since this point is not emphasized in textbooks we give here the derivation [6] of the above statement for the two-level system studied in Section 3. Consider the Bethe-Lamb equations (7) for two states $|a\rangle$ and $|b\rangle$ with decay constants $\gamma_{a(b)}, E_{a}-E_{b}=\hbar \omega_{a}-\hbar \omega_{b}=\hbar \omega$, under influence of the radiation field $\vec{E}(t)=\vec{E}_{0} \sin \nu t$ with Hamiltonian in the dipole form: $H=-e \vec{E} \cdot \vec{r}$, we have:

$$
\begin{aligned}
& \dot{a}=-\frac{\gamma_{a}}{2} a+\frac{i}{\hbar} e \vec{E}_{0} \cdot \vec{r}_{a b} \sin \nu t e^{i \omega t} b \\
& \dot{b}=-\frac{\gamma_{b}}{2} b+\frac{i}{\hbar} e \vec{E}_{0} \cdot \vec{r}_{b a} \sin \nu t e^{i \omega t} a
\end{aligned}
$$

$\left(\vec{r}_{a b}=\langle a|\vec{r}| b\rangle\right)$. These equations are derivable from the Schrödinger equation 


$$
i \hbar \frac{\partial \psi}{\partial t}=\left[H_{0}-\frac{1}{2} i \hbar \Gamma-e \vec{E}(t) \cdot \vec{r}\right] \psi
$$

where $H_{0}$ is the unperturbed Hamiltonian $H_{0}=\frac{\vec{p}}{2 m}+U(\vec{r})$ with eigenstates $\psi_{a}$ and $\phi_{b}$ :

$$
H_{0} \psi_{a}=\hbar \omega_{a} \psi_{a}, H_{0} \psi_{b}=\hbar \omega_{b} \psi_{b}
$$

and $\Gamma$ is the Weisskopf-Wigner type decay operator with eigenvalues $\gamma_{a}, \gamma_{b}$ :

$$
\Gamma \psi_{a(b)}=\gamma_{a(b)} \psi_{a(b)}
$$

Obviously, if we insert the wave function of our two-level system

$$
\psi(\vec{r}, t)=a(t) e^{-i \omega_{a} t} \psi_{a}(\vec{r})+b(t) e^{-i \omega_{b} t} \psi_{b}(\vec{r})
$$

into (B5) we obtain the Bethe-Lamb equations (B4).

Now, to replace the $\vec{E} \cdot \vec{r}$ term in (B5) by the $\vec{p} \cdot \vec{A}$-type interaction (B1) and we make the transformation (B2):

$$
\psi(\vec{r}, t)=T^{+}(\vec{r}, t) \phi(r, t)
$$

with $T(\vec{r}, t)=e^{\frac{i e}{\hbar} \vec{A}(t) \cdot \vec{r}}$ (we retain here $\hbar$ ), thus obtaining the equation for $\phi$ :

$$
i \hbar \frac{\partial \phi}{\partial t}=\left\{H_{0}-\frac{i \hbar}{2} \Gamma^{\prime}(r, t)-\frac{e}{m} \vec{p} \cdot \vec{A}+\frac{e^{2}}{2 m} \vec{A}^{2}\right\} \phi
$$

Thus, we arrive at the important result that in the equivalence stated above between the two forms of interactions (in dipole approximation) it is necessary to treat the transformed damping operator $\Gamma^{\prime}$

$$
\Gamma^{\prime}=T \Gamma T^{+}
$$


In contrast to equations (B4), $\Gamma^{\prime}$ depends on $\vec{r}$ and $t$ and is not diagonal with respect to the eigenstates $\psi_{a(b)}$ of $H_{0}$. Indeed, we have:

$$
\begin{gathered}
\Gamma=\left(\begin{array}{cc}
\gamma_{a} & 0 \\
0 & \gamma_{b}
\end{array}\right) \\
\Gamma^{\prime}=\left(\begin{array}{cc}
\gamma_{a} \cos ^{2} \phi+\gamma_{b} \sin ^{2} \phi & -i\left(\gamma_{a}-\gamma_{b}\right) \sin \phi \cos \phi \\
i\left(\gamma_{a}-\gamma_{b}\right) \sin \phi \cos \phi & \gamma_{a} \sin ^{2} \phi+\gamma_{b} \cos ^{2} \phi
\end{array}\right),
\end{gathered}
$$

where $\phi=e \vec{A}(t) \cdot \frac{\vec{r}_{b a}}{\hbar}$.

These results show that it is wrong to simply replace the $\vec{E} \cdot \vec{r}$ interaction by the $\vec{p} \cdot \vec{A}$ form; the damping matrix has to be changed in addition. The off-diagonal elements of the new damping matrix $\Gamma^{\prime}$ contribute both to the coupling of the $\psi_{a}$ and $\psi_{b}$ states. Finally, it is instructive to write the BetheLamb type equations for the amplitudes $\alpha$ and $\beta$ in the expansion $\phi$ in terms of $\psi_{a}$ and $\psi_{b}$ :

$$
\begin{aligned}
& \dot{\alpha}=-\left(\frac{1}{2} \Gamma_{a a}^{\prime}(t)+\frac{i}{\hbar} \frac{e^{2}}{2 m} \vec{A}^{2}(t)\right) \alpha-\left(\frac{1}{2} \Gamma_{a b}^{\prime}(t)-\frac{i}{\hbar} \frac{e}{m} \vec{p}_{a b} \cdot \vec{A}(t)\right) e^{i \omega t} \beta \\
& \dot{\beta}=-\left(\frac{1}{2} \Gamma_{b b}^{\prime}(t)+\frac{i}{\hbar} \frac{e^{2}}{2 m} \vec{A}^{2}(t)\right) \beta-\left(\frac{1}{2} \Gamma_{b a}^{\prime}(t)-\frac{i}{\hbar} \frac{e}{m} \vec{p}_{b a} \cdot \vec{A}(t)\right) e^{i \omega t} \alpha,
\end{aligned}
$$

where $\vec{p}_{a b}=\langle a|\vec{p}| b\rangle$.

We note that in the calculations of the probability amplitudes in the two representations the initial conditions for the equations of motion (B14) are in general different from the initial conditions of the Bethe-Lamb equations (B4) 6].

A last remark concerns a practical issue. For higher order processes it becomes more and more cumbersome to solve the $\vec{p} \cdot \vec{A}$ equations (B14) due to the transformed damping matrix elements. Therefore, for practical calculations even for a two-level atom the use of the Bethe-Lamb equations (B4) is more convenient. 


\section{Notation Index}

$\lambda_{L}$ - laser radiation wave length measured in $\mathrm{nm}$ or $\mathrm{cm}$

$\lambda_{L}=\frac{\lambda_{L}}{2 \pi}$

$\Delta \lambda_{L}$ - laser radiation linewidth

$\hbar \omega_{L}$ - energy of laser photon measured in $\mathrm{eV}$

$\lambda_{a b} \equiv \frac{2 \pi \hbar c}{E_{a}-E_{b}}$

$\omega_{L}$ - frequency of laser photon

$k_{L}$ - wave number of laser photon

$R_{A}$ - nuclear radius

$\Delta E_{A}$ - nuclear level spacing

$H$ - Hamiltonian

$\alpha=\frac{e^{2}}{\hbar c}$ - fine structure constant

$\vec{R}$ - radius-vector of atomic electron

$\vec{r}_{p}$ - radius-vector of the p-th nuclear proton

$\vec{E}_{L}$ - electric field of laser radiation

$\vec{E}_{0}$ - amplitude of laser electric field

$I$ - laser beam intensity measured in units of $\frac{W}{\mathrm{~cm}^{2}}$

$\phi$ - photon flux

M - matrix element

$\sigma-$ cross section

$\gamma$ - level-decay rate

$\Gamma=\hbar \gamma-$ level width

$N$ - number of laser photons

$|I\rangle$ - initial nuclear state

$|F\rangle$ - final nuclear state

$|N\rangle$ - intermediate nuclear state

$E_{I}$ - energy of initial nuclear state

$E_{F}$ - energy of final nuclear state

$E_{N}$ - energy of intermediate nuclear state

$|i\rangle,|n\rangle,|f\rangle$ - initial, intermediate, and final atomic electronic state, respectively

$\epsilon_{i}, \epsilon_{n}, \epsilon_{f}$ - energy of initial, intermediate, and final atomic electrons, respectively

$E_{I F} \equiv E_{I}-E_{F}$

$\epsilon_{i f} \equiv \epsilon_{i}-\epsilon_{f}$

$E_{i s}$ - energy of nuclear isomeric states 
EB - electron bridge

IEB - inverse electron bridge

IC - internal conversion

$\Delta E$ - nuclear level energy difference

$\Delta \epsilon$ - atomic electron level energy difference

$m(M)$ - electron (proton) mass

$Z$ - atomic number

$Z_{\text {eff }}$ - effective atomic number

$E_{B}$ - electron binding energy in atomic shell, sometimes we use $\left|E_{B}\right| \equiv B$

$c$ - speed of light

$\vec{A}$ - vector potential of electromagnetic field

$\rho\left(\epsilon_{f}\right)$ - density of final electron states

$L$ - multipolarity of nuclear radiation

$l$ - orbital angular momentum

$m$ - magnetic quantum number

$n$ - principal quantum number

$\bar{P}$ - laser average power

$\bar{J}$ - laser average current

$\hat{P}$ - laser peak power

$\rho$ - number density of laser photons

$r_{0}=\frac{e^{2}}{m c^{2}}=2.8 \cdot 10^{-13}$ - classical electron radius

$\lambda_{c}=\frac{\hbar}{m c}=3.86 \cdot 10^{-11} \mathrm{~cm}$ - electron Compton wavelength

$a_{B}=\frac{\hbar^{2}}{m e^{2}}=5.3 \cdot 10^{-9} \mathrm{~cm}-$ Bohr radius

$R_{y}=\frac{e^{2}}{2 a_{B}}-$ Rydberg potential

$\vec{p}=\hbar \vec{k}$ - electron momentum

$\vec{k}$ - wave vector

$E(M) L$ - electric (magnetic) transition with multipolarity $L$

$J_{I(F)}\left(J_{i(f)}\right)$ - nuclear (atomic) angular momentum for initial (final) states

$m_{I(F)}\left(m_{i(f)}\right)$ - nuclear (atomic) magnetic quantum numbers for initial (final)

states

$\Delta$ - energy mismatch; detuning

$T$ - time interval, temperature

$\alpha_{I C}-$ coefficient of internal comversion

$\alpha_{d}$ - coefficient of IC for discrete final electron state

$n, j, \lambda$ - quantum numbers of hydrogen-type solution

$\vec{e}_{1}, \vec{e}_{2}, \vec{e}_{3}$ - unit vectors defining the photon polarization 


\section{References}

[1] F.R. Arutyunyan and V.A. Tumanyan, ITEP Preprint ITEF-137, 1962 (unpublished); Phys.Lett., $\underline{4}$ (1963) 176; JETP, 44 (1963) 2100 (Sov. Phys. JETP, 17 (1963) 1412);

R.H. Milburn, Phys. Rev. Lett. 10 (1963) 75;

F.R. Arutyunyan, I.I. Goldman, and V.A Tumanyan, JETP, 45 (1963) 312 (Sov. Phys. JETP, 18 (1964) 218).

[2] T. Carman, V. Litvinenko, J. Madey, C. Neuman, B. Norum, P. O'Shea, N.R. Roberson, C.Y. Scarlet, E. Schreiber, and H.R. Weller, Nucl. Instr. and Meth. A378 (1996) 1.

[3] A. Einstein, Collected Scientific Works, volume 4, Nauka, Moscow (1967), p.114 (in Russian).

[4] P. Kálmán, Acta Physica Hungarica, 71 (1992) 77.

[5] M. Göppert-Mayer, Naturwiss, 17 (1929) 932;

Ann. Phys. (Leipzig), 9 (1931) 213.

[6] W.E. Lamb, R.R. Schlicher, and M.O. Scully, Phys. Rev. A36 (1987) 2763.

[7] I.S. Batkin, Sov. J. Nuclear Phys. 29 (1979) 464;

ibid, 32 (1980) 972.

[8] I.M. Band, M.A. Listergarten, and A.P. Feresin, Anomalii v koeffitsientakh vnutrenneii konversii $\gamma$-luchei (Anomalies in gamma-ray internal conversion coefficients), Nauka, Leningrad, 1976.

[9] B.S.Dzhelepov, L.K. Peker, and V.O. Sergeev, Skhemy raspada radioaktivnykh yader (Decay schemes of radioactive nuclei), AN SSSR, 1963.

[10] J.A. Bearden and A.F. Burr, Revs. Mod. Phys. 39 (1967) 125.

[11] W. Becker, R.R. Schlicher, M.O. Scully, M.S. Zubairg, and M. Goldhaber, Phys. Lett. 131B (1983) 16.

[12] M.R. Reiss, Phys. Rev. C27 (1983) 1199 1299; C28 (1983) 1402.

[13] J.L. Friar and M.R. Reiss, Phys. Rev. C36 (1987) 283. 
[14] I.M. Ternov, V.N. Rodionov, and O.F. Dorofeev, Fiz. Elem. Chastits At. Yadra, 20 (1989) 51.

[15] L.M. Folan and V.I. Tsifrinovich, Phys. Rev. Lett. 74 (1995) 499.

[16] I.S. Batkin, Yu. G. Smirnov, and T.A. Churakova, Sov. J. Nucl. Phys. 26 (1977) 16.

[17] H.A. Bethe, in Quantentheorie, vol 24/1 of Handbuch der Physik, 2nd ed. (Springer, Berlin, 1933), p. 273.

[18] M. Sargent, M.O. Scully, and W.E. Lamb, Jr., Laser Physics (AddisonWesley, Reading, MA, 1974).

[19] W. Becker, R.R. Schlicher, and M.O. Scully, Phys. Lett. 106A (1984) 441.

[20] V. Weisskopf and E. Wigner, Z. Phys. 63 (1930) 54.

[21] G.C. Baldwin and S.A. Wender, Phys. Rev. Lett. 48 (1982) 1461.

[22] J.F. Berger, D.M. Gogny, and M.S. Weiss, Phys. Rev. A43 (1991) 455; L.C. Biedenharn, G.C. Baldwin, K. Boyer, and J.C. Solem, in Advances in Laser Science I (Univ. of Texas, Dallas, 1985); ed. W.C. Stwalley and M.Lapp (AIP, New York, 1986), p.52;

G.A. Rinner, J.C. Solem, and L.C. Biedenharn, Soc. Photo. Opt. Instrum. Eng. 875 (1988) 92;

J.C. Solem, J. Quant. Spectrosc. Radiat. Transfer, 40 (1988) 713;

F.X. Hartmann, D.W. Noid, and Y.Y. Sharon, Phys. Rev. A44 (1991) 3210 .

[23] P. Kálmán and J. Bergon, Phys. Rev. C34 (1986) 1024.

[24] P. Kálmán, Phys. Rev. C37 (1988) 2676.

[25] P. Kálmán, Phys. Rev. C39 (1989) 2452.

[26] B.A. Zon and F.F. Karpeshin, Zh. Eksp. Teor. Fiz. 70 (1990) 224.

[27] F.F. Karpeshin, I.M. Band, M.B. Trzhaskowskaya, and B.A. Zon, Phys. Lett. B282 (1992) 267. 
[28] D.M. Volkov, Z. Phys. 94 (1935) 250.

[29] J.M. Blatt and V.F. Weisskopf. Theoretical Nuclear Physics, chapter XII (Springer Verlag, New York, 1979).

[30] L.S. Brown and T.W.B. Kibble, Phys. Rev. 133A (1964) 705.

[31] A.I. Nikishov and V.I. Ritus, Zh. Eksp. Teor. Fiz. 46 (1964) 776.

[32] L.V. Keldysh, Sov. Phys. JETP, 20 (1965) 1307.

[33] H.H. Nickle, J. Math. Phys. 7 (1966) 1497.

[34] F. Ehlotzky, Opt. Comm. 27 (1978) 65.

[35] J. Bergon and S. Varró, J. Phys. A14 (1980) 3553.

[36] I.I. Sobelman, Atomic Spectra and Radiative Transitions, volume 1 of Springer Series in Chemical Physics (Springer, Berlin, 1979).

[37] P. Kálmán, Phys. Rev. A39 (1989) 3200.

[38] K.C. Kulander, K.J. Shafer, and J.L. Krause, Phys. Rev. Lett. 66 (1991) 2601.

[39] M. Dörr et al., J. Phys. B26 (1993) L275.

[40] ed. C.M. Lederer and V.S. Shirley, Tables of Isotopes, (Wiley, New York, 1978) 7 th ed.

[41] R.F. O'Connell and C.O. Carroll, Phys. Rev. 138 (1965) 1042.

[42] V.S. Letokhov and C.P. Chebotayev, Nonlinear Laser Spectroscopy, vol. 4 of Springer Series in Optical Sciences, chapter 10, section 2.3, pp. 428-431 (Springer, Berlin, 1977).

[43] V.A. Krutov, Izv. Akad. Nauk SSSR, Ser. Fiz. 22 (1958) 162.

[44] V.A. Krutov and V.N. Fomenko, Ann. Phys. (Leipzig), 21 (1968) 291.

[45] B. Crasemann, Nucl. Instr. Methods 112 (1973) 33.

[46] D. Kekez, A. Ljubičič, K. Pisk, and B.A. Logan, Phys. Rev. Lett. 55 (1985) 1366. 
[47] V.M. Kolomietz and V.N. Kondrat'ev, Sov. J. Nucl. Phys. 51 (1990) 400.

[48] V.A. Zheltonozhskii, P.N. Muzalev, A.F. Novgorodov, and M.A. Ukhin, Sov. Phys. JETP 67 (1988) 10.

[49] P. Kálmán, Phys. Rev. A43 (1991) 2603.

[50] D. Hinneburg, Z. Phys. A300 (1981) 129.

[51] P. Kálmán and T. Keszthelyi, Phys. Rev. A44 (1991) 4761.

[52] V.F. Strizhov and E.V. Tkalya, Sov. Phys. JETP 72 (1991) 387.

[53] L.D. Landau and E.M. Lifshitz, Quantum Mechanics (Pergamon Press, Oxford, 1977).

[54] P. Kálmán and T. Keszthelyi, Phys. Rev. A47 (1993) 1320.

[55] M.R. Shmorak, Nuclear Data Sheets 69 (1993) 375.

[56] M. Morita, Progr. Theor, Phys. 49 (1973) 1574.

[57] E.V. Tkalya, Dokl. Anak. Nauk. SSSR 315 (1990) 1373.

[58] R.V. Arutyunyan and C.A. Bol'shov, Dokl. Anak. Nauk. SSSR 305 (1989) 839.

[59] Y. Izawa and C. Yamanaki, Phys. Lett. 88B (1979) 59.

[60] V.I. Goldanskii and V.A Namiot, JETP Lett. 23 (1976) 451.

[61] V.I. Goldanskii and V.A Namiot, Phys. Lett. 62B (1976) 393.

[62] V.I. Goldanskii and V.A Namiot, Sov. J. Nucl. Phys. 33 (1981) 169.

[63] C.W. Reich and R.G. Helmer, Phys. Rev. Lett. 64 (1990) 271; see also C.W. Reich et al., Int. J. Appl. Radiat. Isot. 35 (1984) 185.

[64] L.A. Kroger and C.W. Reich, Nucl. Phys. A259 (1976) 29; L.A. Kroger, PhD thesis, University of Wyoming (1971) (unpublished).

[65] K.S. Toth, Nucl. Data Sheets 24 (1978) 263. 
[66] D.G. Burke et al., Phys. Rev. C42 (1990) 499.

[67] Y.A. Akovali, Nucl. Data Sheets 58 (1989) 555.

[68] R.G. Helmer and C.W. Reich, Phys. Rev. C49 (1994) 1845.

[69] S. Typel and C. Leclercq-William, Phys. Rev. A53 (1996) 2547.

[70] K.N. Huang et al., At. Data Nucl. Data Tables 18 (1976) 243.

[71] E.V. Tkalya, Pis'ma Zh. Eksp. Theor. Fiz. 55 (1992) 216; JETP Lett. 55 (1992) 211.

[72] E.V. Tkalya, Yad. Fiz. 55 (1992) 2881; Sov. J. Nucl. Phys. 55 (1992) 1611; Nucl. Phys. A539 (1992) 203.

[73] A.I. Akhiezer and V.B. Berestetcskii, Quantum Electrodynamics (Wiley and Sons, New York, 1965).

[74] J.M. Eisenberg and W. Greiner, Excitation Mechanisms of the Nucleus, vol. 2 of Nuclear Theory (North-Holland, Amsterdam, 1970).

[75] V.B. Berestetskii, E.M. Lifshitz, and L.P. Pitaevskii, Quantum Electrodynamics, 2nd ed. (Pergamon Press, Oxford, 1982).

[76] C.H. Corliss and U. Borman, Transition probabilities and oscillator strengths of 70 elements, p.416 (Mir, Moscow, 1968). (in Russian).

[77] A. Giachetti et al., J. Res. Nat. Bur. Stand A78 (1974) 247.

[78] A.A. Radtsig and B.M. Smirnov, Properties of Atoms and Atomic Ions (Energoatomizdat, Moscow, 1986) (in Russian).

[79] P. Kálmán and T. Keszthelyi, Phys. Rev. 49C (1994) 324.

[80] J. Eisenberg and W. Greiner, Nuclear Models, vol. 1 of Nuclear Theory, p.24 (North-Holland, Amsterdam, 1970).

[81] J. Eberly, in Progress in Optics, ed. E. Wulf (North-Holland, Amsterdam, 1969), vol. VII.

[82] J.F. Barger, D. Gogny, and M.S. Weiss, J. Quantum Spectrosc. Radiat. Transfer 40 (1988) 717. 
[83] J.C. Solem and L.C. Biedenharn, J. Quant. Spectrosc. Radiat. Transfer 40 (1988) 707.

[84] G.A. Rinker, J.C. Solem, and L.C. Biedenharn, Soc. Photo-Opt. Instr. Eng. 875 (1988) 92.

[85] G.A. Rinker, J.C. Solem, and L.C. Biedenharn, Advances in Laser Science 1 (1987) 75.

[86] N.B. Delone and V.P. Krainov, Springer Series in Chemical Physics, Atoms in Strong Light Fields, vol. 28 (Springer-Verlag, Berlin- Heiderberg, 1985). 


\section{Figures}

Fig. 1 Feynman diagrams describing the Compton excitation of a nucleus.

Fig. 2 Diagrams describing the "Compton" effect on the bound electron (IEB mechanism). 
Fig. 3 Scheme of an Anti-Stokes $\beta$-transition on a nucleus.

Fig. 4 Scheme of an Anti-Stokes electromagnetic transition on a nucleus. 
Fig. 5 The quantity $T\left(\beta, \frac{\Delta}{\hbar \omega_{L}}\right)$ defined by Eq. (25) (circular polarization) is defined by the relation $I=8.73 \cdot 10^{11} \beta_{0}^{2}$. The curves a-d correspond to $\hbar \omega_{L}=5 \mathrm{eV}$. The nuclei ${ }^{235} \mathrm{U}$ and ${ }^{183} \mathrm{~W}$ are denoted by their electronic shell (see Table 2). The curves $\mathrm{c}$ and $\mathrm{g}$ denoted by $\mathrm{K}$ correspond to the ${ }^{105 m} \mathrm{Ag}$ (K shell) [24].

Fig. 6 Feynman diagrams corresponding to the Electron Bridge mechanism. 
Fig. 7 (a) The spectrum obtained after subtraction of contributions from room background, source impurities, and external bremsstrahlung. The full circles represent experimental data while the full line represents the total estimated spectrum $N\left(E_{\gamma}\right)$. The dashed line represents the ICE contribution in the region of interest; at lower energies there are only ICE contributions. (b) The spectrum produced by the inelastic-electronic-bridge effect with 28.2 $\mathrm{keV}$ photons [46]. 
Fig. 8 Scheme explaining the elimination of the mismatch between the atomic and nuclear level energy differences by laser radiation.

Fig. 9 Feynman diagram describing a laser-assisted resonance deexcitation of a nucleus. 
Fig. $10 \quad F_{K}$ vs. $I$. (a) Two photon case $\left(\hbar \omega_{L}=2.32 \mathrm{eV}\right)$, (b) Four photon case $\left(\hbar \omega_{L}=1.32 \mathrm{eV}\right)$. Curves in both figures are numbered by K [51].

Fig. $11 f_{K}$ vs $K$. (a) and (b) indicate different channel structure of $f_{K}$ at $I=10^{11.5} \frac{\mathrm{W}}{\mathrm{cm}^{2}}$ and $10^{2} \frac{\mathrm{W}}{\mathrm{cm}^{2}}$.51]. 
Fig. 12

(a) Scheme of laser-assisted resonance excitation of a nucleus.

(b)Feynman diagram corresponding to the scheme of Fig. 14(a).

Fig. 13 
Fig. 14 The effective Feynman diagram describing the laser-assisted resonant nuclear excitation.

Fig. 15 A "dressed" (by laser radiation) intermediate electronic state.

Fig. 16 Scheme of the two lowest levels of the thorium-229 nucleus. 
Fig. 17 Deexcitation probability for the $\frac{3}{2}^{+}$state in the ${ }^{229} \mathrm{Th}$ nucleus for the $7 s_{\frac{1}{2}} \rightarrow 8 s_{\frac{1}{2}}$ electronic transitions as a function of laser intensity. The photon energy is fixed to the energy of a resonant two-photon process for a nuclear excitation energy of $3.5 \mathrm{eV}$ (solid line), $4.0 \mathrm{eV}$ (long-dashed line), and $3.0 \mathrm{eV}$ (short-dashed line) or the resonant four-photon process for an energy of $3.5 \mathrm{eV}$ (dotted line) [69]. 
Fig. 18 Scheme of the laser-assisted energy and angular momentum transfer from the atomic shell to the ${ }^{229} \mathrm{Th}$ nucleus. 